\title{
On the Achievable Degrees of Freedom of Two-Cell Multiuser MIMO Interference Networks
}

\author{
Hsin-Jui Chou, Student Member, IEEE, Che-Chen Chou, Student Member, IEEE, \\ Jen-Ming Wu, Member, IEEE, and Ronald Y. Chang, Member, IEEE
}

\begin{abstract}
In this paper, we study the sum degrees of freedom (DoF) of an uplink two-cell multiuser MIMO interference network with asymmetric number of users in the cells. The achievable DoF is devised based on a two-dimensional space-time spreading code framework with linear precoding/decoding design and finite channel extension. The derivation of the achievable DoF is shown related to a rank minimization problem, which corresponds to the minimization of the dimension of the interference subspace. The problem is solved by the proposed grouping algorithm (GA) based on aligning interfering signals into a low-dimensional subspace as a group and attaining the minimum number of groups. The achievable sum DoF derived based on the proposed GA is shown to be greater than prior arts and achieves the theoretic upper bound in several cases. We also give a closed-form expression of the maximum achievable sum DoF when there is the maximum number of admissible users in the considered finite diversity environment.
\end{abstract}

Index Terms-Degrees of freedom, interference alignment, multiuser MIMO, cellular networks, grouping algorithm.

\section{INTRODUCTION}

$\mathbf{I}$ $\mathrm{N}$ multiuser wireless communications, the received signal space constitutes two subspaces, i.e., the (desired) signal subspace and the interference subspace. The sum of the dimension of the two subspaces is a constant specified by system configurations such as antenna numbers at the transmitter and the receiver. A smaller dimension of the interference subspace therefore leads to a greater dimension of the signal subspace. Interference alignment (IA), first introduced by Maddah-Ali et al. [1] as well as Cadambe and Jafar [2], is based on the idea of aligning multiple interfering signals into a subspace of provably smallest dimension such that the dimension of the signal subspace is increased as much as possible for the

Manuscript received November 5, 2013; revised March 31, 2014 and June 21, 2014; accepted June 21, 2014. Date of publication July 1, 2014; date of current version August 20,2014. This work was supported by the National Science Council of Taiwan under Grants NSC 102-2218-E-001-001 and NSC 101-2221-E-007-097. The associate editor coordinating the review of this paper and approving it for publication was H. Dai.

H.-J. Chou is with the Institute of Communications Engineering, National Tsing Hua University, Hsinchu 30013, Taiwan, and also with the Research Center for Information Technology Innovation, Academia Sinica, Taipei 115, Taiwan (e-mail: staray518@gmail.com).

C.-C. Chou and J.-M. Wu are with the Institute of Communications Engineering, National Tsing Hua University, Hsinchu 30013, Taiwan (e-mail: jesse.chou313@gmail.com; jmwu@ee.nthu.edu.tw).

R. Y. Chang is with the Research Center for Information Technology Innovation, Academia Sinica, Taipei 115, Taiwan (e-mail: rchang@ citi.sinica.edu.tw).

Color versions of one or more of the figures in this paper are available online at http://ieeexplore.ieee.org.

Digital Object Identifier 10.1109/TCOMM.2014.2334310 multiuser environment, i.e., the degrees of freedom $(D o F)$ is maximized [1]-[22]. The DoF has been adopted in informationtheoretic studies as the figure of merit that captures the pre$\log$ value of the capacity in high signal-to-noise ratio (SNR) regime. Conceptually, the DoF specifies the maximum supportable number of concurrent interference-free data streams or equivalently the maximum multiplexing gain of the channel [23], [24]. The DoF of various types of multiuser interference channels has been analyzed based on the concept of IA. Jafar and Fakhereddin [3] derived a closed-form expression of DoF for the two-user MIMO interference channel. Gou and Jafar [4] as well as Ghasemi et al. [5] presented a theoretical analysis of inner and outer bounds of the number of DoF for the $K$-user MIMO interference channel. Iterative distributed algorithms were proposed for precoding and decoding designs with the objective of minimizing the interference leakage on the signal subspace for MIMO interference networks [6]-[10]. The feasibility of linear IA for the MIMO interference channel was studied in [8], [25], [26].

The DoF in wireless cellular networks has also been studied. In cellular networks, the same frequency spectrum is reused in multiple geographic areas or cells due to the scarcity of the available spectrum. The received signal therefore contains two types of interference, i.e., inter-cell interference (ICI) and inter-user interference (IUI), which refer respectively to the interference induced when users in the adjacent cells and in the same cell occupy the same spectrum. ICI and IUI have been shown to be the performance-limiting factors in wireless cellular networks [27]. Suh and Tse [12] developed an interference management scheme based on IA which aligns multiple ICI in a one-dimensional subspace and extracts the desired signal from the IUI using a zero-forcing (ZF) based precoding and decoding design. It was also shown in [12] that $K /(K+1)$ DoF per cell can be achieved for a two-cell $K$-user cellular network where users and base stations (BS) are each equipped with a single antenna. The DoF for multiantenna cellular networks where each user has $M$ antennas and each BS has $N$ antennas has also been derived. Park and Lee [13], [14] compared the DoF values between multiple-input single-output (MISO) interfering broadcast channels (IBC) and MIMO interference channels. Sun et al. [15] showed that $K N /(K+\min \{N, K\})$ DoF can be achieved for uplink single-input multiple-output (SIMO) interfering multiple access channels (IMAC). Kim et al. [16] showed the achievability of $\min \{2 N, 2 K M, \max (M, N)\}$ DoF in downlink MIMO IBC via a ZF-based IA scheme. Shin et al. [17] considered a twocell network with two active users in each cell and proposed 
an IA algorithm which achieves the optimal $2 M$ DoF for the case of $[(3 / 2) M\rceil \leq N \leq 2 M$. Sridharan and Yu [18], [19] characterized the DoF of two-cell MIMO cellular networks as a piecewise linear function with either $M$ or $N$ being the bottleneck for both two-user-per-cell and three-user-per-cell cases, following insights from the subspace alignment approaches in [20], [21].

While several outer bounds and achievable schemes for the DoF in wireless cellular networks are known [12]-[19], [22], an exact characterization of the DoF for the case of arbitrary number of users in each cell remains unsolved. In this paper, we derive the achievable sum DoF for the uplink transmission of a two-cell multiuser MIMO interference network with $T$ transmission time (channel extension) and arbitrary (possibly asymmetric) numbers of users in the two cells. The derived achievable strategy is based on a two-dimensional space-time spreading code (2D-STSC) framework with linear coding design and finite channel extension [28], i.e., a vector space strategy [25]. Our main results are summarized as follows:

1) To derive the achievable sum DoF, we first formulate the problem of minimizing the dimension of the interference subspace as a rank minimization problem and then solve the problem by the proposed grouping algorithm (GA). Based on the idea of IA, the GA corresponds to a procedure that aligns multiple interfering data streams into a low-rank ICI subspace as a group and attains the minimum number of groups with the greatest packing ratios [19]. We explicitly formulate the necessary and sufficient grouping rules for obtaining a feasible solution to the rank minimization problem (Theorem 1). The derived achievable sum DoF based on the GA is greater than existing results [16], [17] and achieves the upper bounds derived in [3], [8], [14], [22] in some cases.

2) We derive the achievable sum DoF for a particular case of interest. In our formulation with finite channel extension, the number of users that can simultaneously be served and be allocated at least one dimension of interference-free subspace (i.e., admissible users) is finite. We therefore obtain the maximum achievable sum $\operatorname{DoF}\left(\eta_{\max }\right)$ when there is the maximum number of admissible users in the network. The result is given by a closed-form expression, i.e., $\eta_{\max }=(2 N T-2) / T$ when $N=M$ and $\eta_{\max }=(1 / T)\lfloor(2 \varphi N T) /(\varphi+1)\rfloor$ when $N \geq M$, where $\varphi=\max \{\lfloor(N T-1) /(N T-M T)\rfloor,\lceil(N T+1) / M T\rceil /$ $(\lceil(N T+1) / M T\rceil-1)\}$ (Theorem 2$)$.

The outline of this paper is as follows. In Section II, we present the system model. Problem description is presented in Section III with the proposed solution method given in Section IV. Section V presents the achievable sum DoF results. Numerical verifications and discussions are presented in Section VI. Finally, Section VII concludes the paper.

Notations: Boldface lowercase letters denote vectors and boldface uppercase letters denote matrices. $(\cdot)^{\top}$ denotes the transpose operation. $\mathbf{A}=\left(\mathbf{A}_{1}, \ldots, \mathbf{A}_{K}\right)$ or $\mathbf{A}=\left\{\mathbf{A}_{l}\right\}_{l=1, \ldots, K}$ represents that matrix $\mathbf{A}$ is a horizontal concatenation of matrices $\mathbf{A}_{1}, \ldots, \mathbf{A}_{K} \cdot \operatorname{rank}(\mathbf{A}), \operatorname{vec}(\mathbf{A}), \operatorname{null}\{\mathbf{A}\}, \operatorname{col}\{\mathbf{A}\}$, and $\|\mathbf{A}\|$ denote the rank, the vectorization, the null space, the column space, and the Frobenius norm of matrix A, respectively. $\operatorname{diag}(\cdot)$ represents a block diagonal matrix formed by its elements. $\mathbf{0}_{m \times n}$ denotes an $m \times n$ zero matrix. $\mathbb{N}, \mathbb{N}_{+}$, and $\mathbb{R}$ represent the set of nonnegative integers, positive integers, and real numbers, respectively. Calligraphic letters denote the sets, and $|\mathcal{A}|$ is the cardinality of set $\mathcal{A} .\lceil\cdot\rceil$ and $\lfloor\cdot\rfloor$ represent the ceiling and floor operations, respectively.

\section{SYSTEM MODEL}

We consider the uplink transmission in a two-cell MIMO interference network with possibly asymmetric numbers of users in the two cells. The $K^{[a]}$ users in the $a$-th cell communicate simultaneously with the base station (BS) in the $a$-th cell over $T$ time slots, $a=1,2$. The BS, with $N$ antennas, and the $k$-th user $\left(k=1, \ldots, K^{[a]}\right)$ in cell $a$, with $M$ antennas, are denoted as $\operatorname{BS}^{[a]}$ and user $(k,[a])$, respectively. We assume $N \geq M$ to reflect practical scenarios. At time $t(t=1, \ldots, T)$, the received signal at $\mathrm{BS}^{[a]}$ can be represented from the perspective of user $(k,[a])$ as

$$
\begin{aligned}
\tilde{\mathbf{y}}^{[a]}(t)=\tilde{\mathbf{H}}_{k}^{[a, a]}(t) \tilde{\mathbf{x}}_{k}^{[a]}(t) & +\underbrace{\sum_{l=1, l \neq k}^{K^{[a]}} \tilde{\mathbf{H}}_{l}^{[a, a]}(t) \tilde{\mathbf{x}}_{l}^{[a]}(t)}_{\text {IUI for user }(k,[a])} \\
& +\underbrace{\sum_{l=1}^{K^{[\bar{a}]}} \tilde{\mathbf{H}}_{l}^{[a, \bar{a}]}(t) \tilde{\mathbf{x}}_{l}^{[\bar{a}]}(t)}_{\text {ICI for user }(k,[a])}+\tilde{\mathbf{z}}^{[a]}(t)
\end{aligned}
$$

where $\tilde{\mathbf{x}}_{k}^{[a]}(t) \in \mathbb{R}^{M \times 1}$ is the transmit signal from user $(k,[a])$ satisfying an average power constraint over $T$ time slots, i.e., $(1 / T) \sum_{t=1}^{T} \mathbb{E}\left[\left\|\tilde{\mathbf{x}}_{k}^{[a]}(t)\right\|^{2}\right] \leq \sigma_{x}^{2} ; \tilde{\mathbf{H}}_{k}^{[a, b]}(t) \in \mathbb{R}^{N \times M}$ is the uplink channel from user $(k,[b])$ to $\mathrm{BS}^{[a]}$ for $a, b \in\{1,2\}$; and $\tilde{\mathbf{z}}^{[a]}(t) \in \mathbb{R}^{N \times 1}$ is the additive white Gaussian noise (AWGN) with zero mean and variance $\sigma_{z}^{2}$. Throughout the paper, we use index $\bar{a}=3-a$ to denote specifically the other cell from the perspective of cell $a$, and indices $a, b \in\{1,2\}$ to denote any possible cell combination. Each entry in $\tilde{\mathbf{H}}_{k}^{[a, b]}(t)$ is independent and identically distributed (i.i.d.) according to some continuous distribution. The channel matrix from different users to the BS are assumed to be independent in the matrix space $\mathbb{R}^{N \times M}$ and are perfectly known at all nodes.

We consider an achievable scheme of interference alignment based on the 2D-STSC structure [28], where each user transmits a precoded signal over $T$ time slots under a time-varying channel. User $(k,[a])$ sends $d_{k}^{[a]} \in \mathbb{N}$ data streams to $\mathrm{BS}^{[a]}$, and the $i$-th $\left(i=1, \ldots, d_{k}^{[a]}\right)$ data stream of user $(k,[a])$ is multiplied by a precoding matrix specific to the $i$-th data stream before transmission. Thus, the transmit signal of user $(k,[a])$ over $T$ time slots can be expressed as

$$
\left[\tilde{\mathbf{x}}_{k}^{[a]}(1), \ldots, \tilde{\mathbf{x}}_{k}^{[a]}(T)\right]=\sum_{i=1}^{d_{k}^{[a]}} s_{k, i}^{[a]} \mathbf{W}_{k, i}^{[a]}
$$




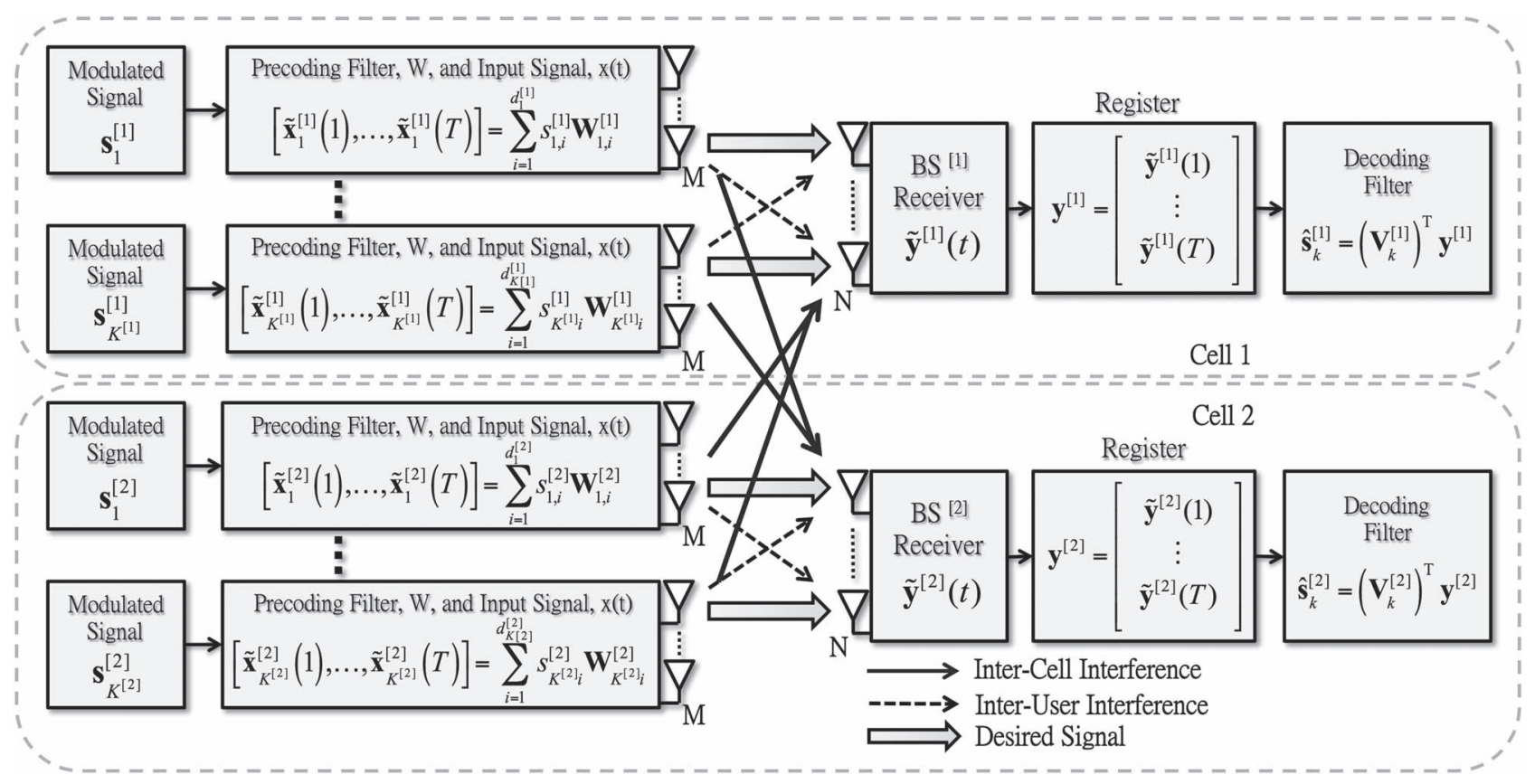

Fig. 1. Block diagram of an uplink two-cell MIMO interference network with 2D-STSC.

where $s_{k, i}^{[a]} \in \mathbb{R}$ and $\mathbf{W}_{k, i}^{[a]} \in \mathbb{R}^{M \times T}$ are the $i$-th data stream of user $(k,[a])$ and the corresponding precoding matrix, respectively. We consider that the data streams for all users are linearly independent and each data stream is decoded individually at the BS. The total received signal at $\mathrm{BS}^{[a]}$ over $T$ time slots is given by (from the perspective of user $(k,[a]))$

$$
\begin{gathered}
\mathbf{y}^{[a]}=\mathbf{H}_{k}^{[a, a]} \mathbf{W}_{k}^{[a]} \mathbf{s}_{k}^{[a]}+\underbrace{\sum_{l=1, l \neq k}^{K^{[a]}} \mathbf{H}_{l}^{[a, a]} \mathbf{W}_{l}^{[a]} \mathbf{s}_{l}^{[a]}}_{\text {IUI for user }(k,[a])} \\
+\underbrace{\sum_{l=1}^{\left.K^{[a]}\right]} \mathbf{H}_{l}^{[a, \bar{a}]} \mathbf{W}_{l}^{[\bar{a}]} \mathbf{s}_{l}^{[\bar{a}]}}_{\text {ICI for user }(k,[a])}+\mathbf{z}^{[a]}
\end{gathered}
$$

where

$$
\begin{aligned}
\mathbf{y}^{[a]} & =\left[\left(\tilde{\mathbf{y}}^{[a]}(1)\right)^{\top}, \ldots,\left(\tilde{\mathbf{y}}^{[a]}(T)\right)^{\top}\right]^{\top} \in \mathbb{R}^{N T \times 1}, \quad(4 \mathrm{a}) \\
\mathbf{H}_{k}^{[a, b]} & =\operatorname{diag}\left(\tilde{\mathbf{H}}_{k}^{[a, b]}(1), \ldots, \tilde{\mathbf{H}}_{k}^{[a, b]}(T)\right) \in \mathbb{R}^{N T \times M T}, \quad(4 \mathrm{~b}) \\
\mathbf{W}_{k}^{[a]} & =\left[\operatorname{vec}\left(\mathbf{W}_{k, 1}^{[a]}\right), \ldots, \operatorname{vec}\left(\mathbf{W}_{k, d_{k}^{[a]}}^{[a]}\right)\right] \in \mathbb{R}^{M T \times d_{k}^{[a]}}, \\
\mathbf{s}_{k}^{[a]} & =\left[s_{k, 1}^{[a]}, \ldots, s_{k, d_{k}^{[a]}}^{[a]}\right]^{\top} \in \mathbb{R}_{k}^{d_{k}^{[a]} \times 1}, \\
\mathbf{z}^{[a]} & =\left[\left(\tilde{\mathbf{z}}^{[a]}(1)\right)^{\top}, \ldots,\left(\tilde{\mathbf{z}}^{[a]}(T)\right)^{\top}\right]^{\top} \in \mathbb{R}^{N T \times 1} .
\end{aligned}
$$

Note that we consider that a diversity of order $T$ is achieved from the frequency/time diversity of the wireless channel over $T$ time slots [29] by representing $\mathbf{H}_{k}^{[a, b]}$ in a block diagonal form in (4b). As can be seen from (3), the received signal at
$\mathrm{BS}^{[a]}$ contains the useful signal as well as IUI and ICI for user $(k,[a])$. To mitigate the interference in decoding the desired signal for user $(k,[a]), \mathrm{BS}^{[a]}$ multiplies the received signal by a decoding matrix $\mathbf{V}_{k}^{[a]} \triangleq\left[\mathbf{v}_{k, 1}^{[a]}, \ldots, \mathbf{v}_{k, d_{k}^{[a]}}^{[a]}\right] \in \mathbb{R}^{N T \times d_{k}^{[a]}}$. The decoded data stream vector for user $(k,[a])$ is thus given by

$$
\hat{\mathbf{s}}_{k}^{[a]}=\left(\mathbf{V}_{k}^{[a]}\right)^{\top} \mathbf{y}^{[a]} .
$$

The described space-time transmission system is illustrated in Fig. 1.

The information-theoretic sum DoF specifies the number of interference-free signaling dimensions in the spatial domain or equivalently the maximum number of interference-free data streams that can be transmitted concurrently per channel use. The information-theoretic sum DoF is defined as the pre-log factor of the maximum sum rate [2]-[4], i.e.,

$$
\tilde{\eta} \triangleq \limsup _{\rho \rightarrow \infty}\left[\sup _{\tilde{\mathcal{R}}(\rho) \in \mathcal{C}(\rho)} \frac{\sum_{a=1}^{2} \sum_{k=1}^{K^{[a]}} \tilde{R}_{k}^{[a]}(\rho)}{\log (\rho)}\right]
$$

where $\tilde{R}_{k}^{[a]}(\rho)$ denotes the achievable rate of user $(k,[a])$ at SNR $\rho$, and $\mathcal{C}(\rho)$ denotes the capacity region which is the set of all achievable rate tuples $\tilde{\mathcal{R}}(\rho)=\left(\tilde{R}_{1}^{[1]}(\rho), \ldots, \tilde{R}_{K^{[1]}}^{[1]}(\rho)\right.$, $\left.\tilde{R}_{1}^{[2]}(\rho), \ldots, \tilde{R}_{K^{[2]}}^{[2]}(\rho)\right)$. Instead of studying the informationtheoretic notion of DoF where the codewords may span arbitrarily long channel extensions to achieved the theoretic bound, in this paper, we focus on a practical precoding/decoding design scheme (i.e., a 2D-STSC scheme) with given $T$ transmission time (channel extension). The 2D-STSC scheme-specific sum $D o F$, which is also referred to as the spatial multiplexing gain [30] and is defined below, is considered as the figure of merit for our precoding and decoding design. 
Definition 1 (2D-STSC Scheme-Specific Sum DoF): Based on the 2D-STSC structure with system configurations of $\left(K^{[1]}, K^{[2]}, M, N, T\right)$, the 2D-STSC scheme-specific sum DoF for a two-cell MIMO interference network is described by

$$
\eta^{*} \triangleq \limsup _{\rho \rightarrow \infty} \frac{\sum_{a=1}^{2} \sum_{k=1}^{K^{[a]}} R_{k}^{[a]}(\rho)}{\log (\rho)}
$$

where $R_{k}^{[a]}(\rho)$ is the data rate for user $(k,[a])$ at $\operatorname{SNR} \rho$ based on the 2D-STSC structure, as shown in (8), shown at the bottom of the page, where $a$ and $\bar{a}=3-a$ are cell indices, and $\rho=\sigma_{x}^{2} / \sigma_{z}^{2}$ is the SNR.

Note that a specific coding scheme in the considered 2D-STSC structure yields a DoF value that is always nongreater than the theoretic value, i.e., $\eta^{*} \leq \tilde{\eta}$ [25]. For simplicity, in the following discussions we will use "sum DoF" to refer to "2D-STSC scheme-specific sum DoF".

\section{PRoblem Description}

Precoding and decoding designs based on the maximization of the sum rate in (8) using an iterative algorithm have been proposed in [30], [31]. This approach has several disadvantages: 1) the global optimal solution is not guaranteed, 2) the feasibility of the solutions is open for asymmetric cases, and 3 ) the complexity of the iterative method is prohibitively high. In this paper, we propose a precoding and decoding design based on the sum DoF in (7) without iterative computations, and examine the allocation of the sum DoF to users with the consideration of the maximum supportable number of users in a given finite channel diversity of $M N T$.

From Definition 1, the sum rate is dominated by the interference (IUI and ICI) when the SNR goes to infinity. Thus, the interference should be nullified by the decoding matrix in order to maximize the data rate. With the asymptotic rate approximation $R_{k}^{[a]}(\rho)=\left(d_{k}^{[a]} / T\right) \log (\rho)+o(\log (\rho)), \forall k, a$ [32, Chapter 7], where $o(\cdot)$ is the Landau symbol that gives $o(\log (\rho)) / \log (\rho) \rightarrow 0$ as $\rho \rightarrow \infty$, it follows that the sum DoF $\eta^{*}$ specifies the maximum number of interference-free data stream transmission per time slot. Therefore, the sum DoF $\eta^{*}$ for the two-cell MIMO interference network with system configurations of $\left(K^{[1]}, K^{[2]}, M, N, T\right)$ is specified by the solution to the following problem:

$$
\max _{\mathbf{W}_{k}^{[a]}, \mathbf{V}_{k}^{[a]}, \forall a, k} \frac{1}{T} \sum_{a=1}^{2} \sum_{k=1}^{K^{[a]}} d_{k}^{[a]}
$$

$$
\begin{array}{ll}
\text { s.t. } & \mathbf{V}_{k}^{[a]} \subseteq \operatorname{null}\left\{\left(\mathbf{Q}_{\mathrm{IUI}, k}^{[a]}, \mathbf{Q}_{\mathrm{ICI}}^{[a]}\right)^{\top}\right\}, \forall k, a \\
& \operatorname{rank}\left(\left(\mathbf{V}_{k}^{[a]}\right)^{\top} \mathbf{H}_{k}^{[a, a]} \mathbf{W}_{k}^{[a]}\right)=d_{k}^{[a]}, \forall k, a \\
& d_{k}^{[a]} \leq M T, \forall k, a
\end{array}
$$

where

$$
\begin{aligned}
\mathbf{Q}_{\mathrm{IUI}, k}^{[a]} & \triangleq\left\{\mathbf{H}_{l}^{[a, a]} \mathbf{W}_{l}^{[a]}\right\}_{\substack{l=1, \ldots, K^{[a]} \\
l \neq k}} \\
\mathbf{Q}_{\mathrm{ICI}}^{[a]} & \triangleq\left\{\mathbf{H}_{l}^{[a, \bar{a}]} \mathbf{W}_{l}^{[\bar{a}]}\right\}_{l=1, \ldots, K^{[a]}}
\end{aligned}
$$

are equivalent IUI and ICI channels for user $(k,[a])$, respectively. Constraint (10) represents that the decoding matrices project the interference onto the interference subspace which is separated from the desired signal. Constraint (11) ensures that the desired signal vectors at each BS span all the available interference-free subspace. The interference subspace and the interference-free subspace are orthogonal complements of each other at each BS. Constraint (12) represents the fact that the precoding matrix $\mathbf{W}_{k}^{[a]}$ maps the data stream vector $\mathbf{s}_{k}^{[a]}$ to an $M T$-dimensional transmit signal and the number of data streams is upper bounded by $M T$.

The IUI and ICI subspaces for user $(k,[a])$ are spanned by the columns of $\mathbf{Q}_{\mathrm{IUI}, k}^{[a]}$ and $\mathbf{Q}_{\mathrm{ICI}}^{[a]}$, respectively, denoted by $\operatorname{col}\left\{\mathbf{Q}_{\mathrm{IUI}, k}^{[a]}\right\}$ and $\operatorname{col}\left\{\mathbf{Q}_{\mathrm{ICI}}^{[a]}\right\}$. The total interference subspace for user $(k,[a])$ is thus given by $\operatorname{col}\left\{\mathbf{Q}_{k}^{[a]}\right\} \triangleq \operatorname{col}\left\{\mathbf{Q}_{\mathrm{IUI}, k}^{[a]}, \mathbf{Q}_{\mathrm{ICI}}^{[a]}\right\}$. Since (11) stipulates the independency of all desired signal vectors at each BS, the rank of the IUI subspace for the signal from user $(k,[a])$ is the sum of data streams from all other users in cell $a$, i.e., $\operatorname{rank}\left(\mathbf{Q}_{\mathrm{IUI}, k}^{[a]}\right)=\sum_{l=1, l \neq k}^{K^{[a]}} d_{l}^{[a]}$. Also, the IUI and ICI subspaces are linearly independent. Thus, the dimension of $\mathbf{Q}_{k}^{[a]}$ is $N T \times\left(\left(\sum_{l=1, l \neq k}^{K^{[a]}} d_{l}^{[a]}\right)+\operatorname{rank}\left(\mathbf{Q}_{\mathrm{ICI}}^{[a]}\right)\right)$. According to (10), in order to ensure interference-free transmission for user $(k,[a])$, the number of transmit data streams for user $(k,[a])$ should not exceed the nullity of $\left(\mathbf{Q}_{k}^{[a]}\right)^{\top}$, i.e.,

$$
d_{k}^{[a]} \leq N T-\left(\sum_{l=1, l \neq k}^{K^{[a]}} d_{l}^{[a]}\right)-\operatorname{rank}\left(\mathbf{Q}_{\mathrm{ICI}}^{[a]}\right) .
$$

$$
R_{k}^{[a]}(\rho)=\frac{1}{T} \sum_{i=1}^{d_{k}^{[a]}} \log \left(1+\frac{\left\|\left(\mathbf{v}_{k, i}^{[a]}\right)^{\top} \mathbf{H}_{k}^{[a, a]} \operatorname{vec}\left(\mathbf{W}_{k, i}^{[a]}\right)\right\|^{2}}{\sigma_{z}^{2}+\underbrace{\sum_{l=1, l \neq k}^{K^{[a]}}\left\|\left(\mathbf{v}_{k, i}^{[a]}\right)^{\top} \mathbf{H}_{l}^{[a, a]} \mathbf{W}_{l}^{[a]}\right\|^{2}}_{\text {IUI for user }(k,[a])}+\underbrace{\sum_{l=1}^{K^{[a]}}\left\|\left(\mathbf{v}_{k, i}^{[a]}\right)^{\top} \mathbf{H}_{l}^{[a, \bar{a}]} \mathbf{W}_{l}^{[\bar{a}]}\right\|^{2}}_{\text {ICI for user }(k,[a])}}\right)
$$


In addition, from (12) we have

$$
\begin{aligned}
\sum_{k=1}^{K^{[a]}} d_{k}^{[a]} & \leq \min \left\{K^{[a]} M T, N T-\operatorname{rank}\left(\mathbf{Q}_{\mathrm{ICI}}^{[a]}\right)\right\} \\
& \leq \min \left\{K^{[a]} M T, N T-\gamma^{*[a]}\right\}
\end{aligned}
$$

where $\gamma^{*[a]}$ specifies the minimum rank of $\mathbf{Q}_{\mathrm{ICI}}^{[a]}$ (i.e., the minimum dimension of the ICI subspace) and is a function of the number of data streams from the other cell (i.e., cell $\bar{a}$ ). When (15b) is met with equality, according to the problem in (9), the sum DoF can be expressed as

$$
\eta^{*}=\frac{1}{T} \min \left\{\left(K^{[1]}+K^{[2]}\right) M T, 2 N T-\sum_{a=1}^{2} \gamma^{*[a]}\right\} .
$$

Clearly, the smaller the dimension of the ICI subspace, the greater sum DoF will be yielded. The problem of minimizing the dimension of the ICI subspace with given numbers of data streams is formulated as the following rank minimization problem:

$$
\begin{aligned}
& \mathcal{P}: \min _{\mathbf{W}_{1}^{[\bar{a}]}, \ldots, \mathbf{W}_{K}^{[\bar{a}]}} \operatorname{rank}\left(\mathbf{Q}_{\mathrm{ICI}}^{[a]}\right) \\
& \text { s.t. } \quad \operatorname{rank}\left(\mathbf{W}_{k}^{[\bar{a}]}\right)=d_{k}^{[\bar{a}]}, \quad \forall k .
\end{aligned}
$$

The full-rank constraint of $\mathbf{W}_{k}^{[\bar{a}]}$ inherits the constraint in (11) which ensures that the transmit signal of user $(k,[\bar{a}])$ spans all the available interference-free subspace. The optimal solution to Problem $\mathcal{P}$ is specified by $\gamma^{*[a]}$.

Problem $\mathcal{P}$ is challenging to solve for two reasons: 1) the fullrank constraint is defined over a nonconvex set; and 2) even by relaxing the constraint to a convex feasible set with positivedefinite precoding matrices [33], the problem is still a nonconvex optimization problem with exponential running time [34][36]. To solve Problem $\mathcal{P}$, we propose a noniterative algorithm, which is termed the grouping algorithm (GA), based on the concept of IA. The GA produces a feasible solution $\gamma^{[a]}$ to Problem $\mathcal{P}^{1}$ as it provides a low-complexity precoding/decoding design (Section IV). Then, we derive the achievable sum DoF for some precoding/decoding scheme based on the results of the GA, i.e.,

$$
\eta=\frac{1}{T} \min \left\{\left(K^{[1]}+K^{[2]}\right) M T, 2 N T-\sum_{a=1}^{2} \gamma^{[a]}\right\}
$$

where $\eta \leq \eta^{*}$, and $\gamma^{[a]} \geq \gamma^{*[a]}$ is the dimension of the ICI subspace realized by the precoding/decoding scheme (Section V).

\section{Proposed Grouping Algorithm BASED ON INTERFERENCE ALIGNMENT}

In this section, we present the proposed GA which solves Problem $\mathcal{P}$ by dividing all data streams of users in the other cell (cell $\bar{a}$ ) into groups and aligning the ICI signal from the same group into a low-rank ICI subspace at $\mathrm{BS}^{[a]}$. We adopt the concept of packing ratio [19], defined as the ratio between

\footnotetext{
${ }^{1}$ A solution to Problem $\mathcal{P}$ is feasible if it corresponds to a set of precoding matrices that satisfy the full-rank constraint.
}

the number of data streams in a group and the dimension of the ICI subspace spanned by the data streams in this group. For example, if the $i$-th data stream of user $(k,[2])$ and the $j$-th data stream of user $(l,[2])$ are assigned to the same group with packing ratio $2: 1$, we have

$$
\operatorname{col}\left\{\mathbf{H}_{k}^{[1,2]} \operatorname{vec}\left(\mathbf{W}_{k, i}^{[2]}\right), \mathbf{H}_{l}^{[1,2]} \operatorname{vec}\left(\mathbf{W}_{l, j}^{[2]}\right)\right\}=1
$$

where $\mathbf{H}_{k}^{[a, \bar{a}]} \operatorname{vec}\left(\mathbf{W}_{k, i}^{[\bar{a}]}\right)$ represents the equivalent ICI channel corresponding to the $i$-th data stream of user $(k,[\bar{a}])$. In this case, the two equivalent ICI channels are linearly dependent and the dimension of the ICI subspace spanned by the data streams in this group is one.

Consider that the GA divides the data streams into a total of $\zeta \in \mathbb{N}_{+}$groups. Let $\kappa_{g} \in \mathbb{N}_{+}$be the number of data streams in the $g$-th group, where $\sum_{g=1}^{\zeta} \kappa_{g}=\sum_{k=1}^{K^{[\bar{a}]}} d_{k}^{[\bar{a}]}$, and let $r_{g} \in \mathbb{N}$ be the dimension of the ICI subspace spanned by the equivalent ICI channels corresponding to the data streams in the $g$-th group. Then, the packing ratio of the $g$-th group is $\kappa_{g}: r_{g}$ (where $\kappa_{g} \geq r_{g}$ ), and the solution to Problem $\mathcal{P}$ yielded by the GA is given by the relationship

$$
\gamma^{[a]}=\min \left\{\sum_{g=1}^{\zeta} r_{g}, N T\right\} .
$$

Clearly, the greater the packing ratios, the smaller the dimension of the ICI subspace for a given number of data streams.

Define the set of data streams in the $g$-th group with a packing ratio $\kappa_{g}: r_{g}$ as

$$
\begin{gathered}
\mathcal{G}_{g}=\left\{D_{k, i}^{[2]}, D_{l, j}^{[2]}, \ldots, D_{p, q}^{[2]} \mid \operatorname{rank}\left(\operatorname { c o l } \left\{\mathbf{H}_{k}^{[1,2]} \operatorname{vec}\left(\mathbf{W}_{k, i}^{[2]}\right),\right.\right.\right. \\
\left.\left.\left.\mathbf{H}_{l}^{[1,2]} \operatorname{vec}\left(\mathbf{W}_{l, j}^{[2]}\right), \ldots, \mathbf{H}_{p}^{[1,2]} \operatorname{vec}\left(\mathbf{W}_{p, q}^{[2]}\right)\right\}\right)=r_{g}\right\}
\end{gathered}
$$

where $D_{k, i}^{[2]}$ denotes the $i$-th data stream from user $(k,[2]) ; k$, $l, p$ and $i, j, q$ refer to certain users and certain data streams in general, respectively; and $\left|\mathcal{G}_{g}\right|=\kappa_{g}$. The $i$-th data stream from user $(k,[2])$, the $j$-th data stream from user $(l,[2])$, and the $q$-th data stream from user $(p,[2])$ are grouped together in the $g$-th group. There are many possible coding structures or aligning strategies to find the precoding matrices that satisfy (21). However, not every coding structure or aligning strategy will lead to a feasible solution to Problem $\mathcal{P}$. To find a feasible solution we need: 1) a coding structure that produces non-trivial precoding vectors for every data stream in each group, and 2) a proper composition of data streams in each group that ensures the independence of the precoding vectors of each user.

For 1), it is in general difficult to establish a coding structure that produces non-trivial precoding vectors for the data streams in a group with an arbitrary packing ratio, as the problem is related to algebraic geometry and is open in general [8], [22]. Thus, we consider a restricted form of the problem by focusing on two types of packing ratios, namely, $\kappa_{g}: 1$ (where $\kappa_{g} \in \mathbb{N}_{+}$) and $\kappa_{g}: \kappa_{g}-1$ (where $\kappa_{g} \in \mathbb{N}_{+}$and $\kappa_{g} \geq 3$ ), for which we can show that they lead to a feasible solution to Problem $\mathcal{P}$. Note that any arbitrary packing ratio can be treated as a combination of one or both of these two types of packing ratios (e.g., the packing ratio $5: 3$ can be decomposed into $3: 1,1: 1$, 
$1: 1 ;$ or $2: 1,3: 2$; or $2: 1,2: 1,1: 1)$, although the resulting coding structures will lead to suboptimal solutions compared to what would be yielded with a general coding structure. With our consideration, the problem of finding the precoding matrices that satisfy (21) can be described by the following problem of solving $\breve{\mathbf{w}}_{g}$ :

$$
\breve{\mathbf{H}}_{g} \breve{\mathbf{w}}_{g}=\mathbf{0}_{\left(\kappa_{g}-r_{g}\right) N T \times 1}
$$

where

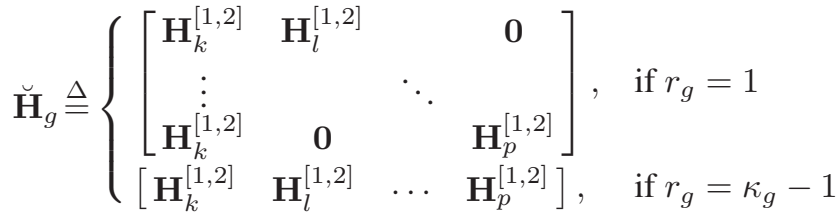

$$
\begin{aligned}
& \breve{\mathbf{w}}_{g} \triangleq\left[\left(\operatorname{vec}\left(\mathbf{W}_{k, i}^{[2]}\right)\right)^{\top},\left(\operatorname{vec}\left(\mathbf{W}_{l, j}^{[2]}\right)\right)^{\top}, \ldots,\left(\operatorname{vec}\left(\mathbf{W}_{p, q}^{[2]}\right)\right)^{\top}\right]^{\top} \text {. }
\end{aligned}
$$

The dimension of $\breve{\mathbf{H}}_{g}$ and $\breve{\mathbf{w}}_{g}$ are $\left(\kappa_{g}-r_{g}\right) N T \times \kappa_{g} M T$ and $\kappa_{g} M T \times 1$, respectively. Clearly, each precoding vector corresponding to the data stream in the $g$-th group lies in the precoding vector set of $\breve{\mathbf{w}}_{g} \subseteq$ null $\left\{\breve{\mathbf{H}}_{g}\right\}$. Consider a general case that the data streams in the $g$-th group are from $\chi_{g}(1 \leq$ $\left.\chi_{g} \leq \kappa_{g}\right)$ different users. Then, the rank of the matrix $\breve{\mathbf{H}}_{g}$ is $\min \left\{\kappa_{g} M T,\left(\kappa_{g}-r_{g}\right) N T\right\}$ for $r_{g}=1$ and $\min \left\{\chi_{g} M T,\left(\kappa_{g}-\right.\right.$ $\left.\left.r_{g}\right) N T\right\}$ for $r_{g}=\kappa_{g}-1$. It is easy to show that we can find a nonzero vector for every precoding vector in the solution set of $\breve{\mathbf{w}}_{g}$ if and only if $\breve{\mathbf{H}}_{g}$ is full rank and has more columns than rows, i.e., the relation $\kappa_{g} M T>\min \left\{\kappa_{g} M T,\left(\kappa_{g}-r_{g}\right) N T\right\}$ holds, which is $\kappa_{g} M T>\left(\kappa_{g}-r_{g}\right) N T$ for $r_{g}=1$ and $\chi_{g} M T>$ $\left(\kappa_{g}-r_{g}\right) N T$ for $r_{g}=\kappa_{g}-1$.

For 2), we establish three grouping rules in the following based on the coding structure in (22) to find a proper composition of data streams in each group. The three grouping rules will be shown to be necessary and sufficient conditions for the GA to produce a feasible solution to Problem $\mathcal{P}$. Then, we present the general procedure of the GA based on the three grouping rules. Without loss of generality, we focus on cell 1's perspective, i.e., $a=1$ and $\bar{a}=2$.

\section{A. Grouping Algorithm: Grouping Rules}

Theorem 1: The GA will produce a feasible solution to Problem $\mathcal{P}$ if and only if the following three grouping rules are all satisfied:

(R.1) The maximum number of data streams from the same user that can be grouped together in the same $g$-th group is $r_{g}$.

(R.2) If the data streams in the $g$-th group are from $\chi_{g}$ different users, the number of data streams that can be grouped together in the $g$-th group is upper bounded by

$$
\kappa_{g} \leq\left\{\begin{array}{cl}
\left\lfloor\frac{\chi_{g} M T+r_{g} N T-1}{N T}\right\rfloor, & \text { if } N>M \\
\infty, & \text { if } N=M .
\end{array}\right.
$$

(R.3) We say two groups are homogeneous when two groups have the same packing ratio and contain the same number

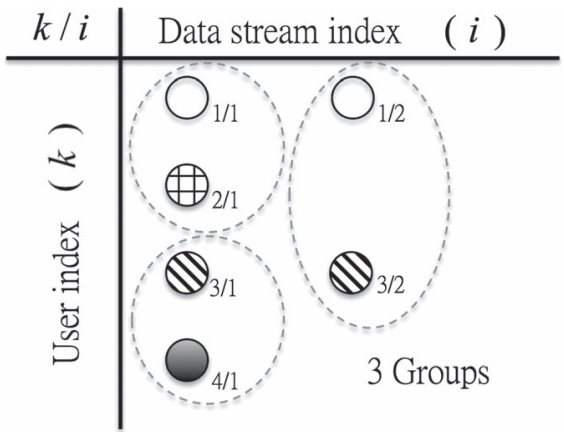

Fig. 2. An exemplary grouping by the proposed GA for a two-cell MIMO interference network with $\left(K^{[2]}, M, N, T\right)=(4,3,5,1)$ and $\left(d_{1}^{[2]}, d_{2}^{[2]}\right.$, $\left.d_{3}^{[2]}, d_{4}^{[2]}\right)=(2,1,2,1)$ in Example 1. Data streams from the same user are shown by the same pattern. A minimum number of $\zeta=3$ groups is yielded. The solution to Problem $\mathcal{P}$ is given by $\gamma^{[1]}=3$.

of data streams all from the same set of users. The maximum number of homogeneous groups is

$$
\psi\left(\kappa_{h}, r_{h}\right)=\left(\kappa_{h} M T-\left(\kappa_{h}-r_{h}\right) N T\right)^{+}
$$

where $\kappa_{h}: r_{h}$ is the packing ratio in each homogeneous group, and $(x)^{+}=\max \{x, 0\}$.

Proof: See the Appendix.

Remark 1: It is useful to consider a more restrictive set of rules in practice to produce a feasible solution to Problem $\mathcal{P}$ efficiently without compromising the optimal solution. Specifically, it can be easily seen that substituting $\chi_{g}=\kappa_{g}$ (meaning the data streams in the $g$-th group are each from a different user) into (R.2) allows the greatest packing ratios and consequently leads to the smallest dimension of the ICI subspace. The alternative rules as a result of setting $\chi_{g}=\kappa_{g}$ are as follows:

(R.1*) Data streams from the same user cannot be grouped together in the same $g$-th group.

(R.2*) The number of data streams that can be grouped together in the $g$-th group is upper bounded by

$$
\kappa_{g} \leq\left\{\begin{array}{cl}
\left\lfloor\frac{r_{g} N T-1}{N T-M T}\right\rfloor, & \text { if } N>M \\
\infty, & \text { if } N=M .
\end{array}\right.
$$

The GA will produce a feasible solution to Problem $\mathcal{P}$ if (R. $1^{*}$ ), (R.2*), and (R.3) are all satisfied (i.e., these three grouping rules are sufficient conditions for the GA to produce a feasible solution to Problem $\mathcal{P}$ ).

Example 1: Consider a two-cell MIMO interference network with system configurations of $\left(K^{[2]}, M, N, T\right)=(4,3,5,1)$ and $\left(d_{1}^{[2]}, d_{2}^{[2]}, d_{3}^{[2]}, d_{4}^{[2]}\right)=(2,1,2,1)$. According to (R.2*), which gives $\kappa_{g} \leq\left\lfloor(1 / 2)\left(5 r_{g}-1\right)\right\rfloor$, we have all possible packing ratios in descending order as follows: $\left(\kappa_{g}: r_{g}\right)=(2: 1)$, $(3: 2),(4: 3),(5: 4),(6: 5),(1: 1)$. Starting from the largest packing ratio $2: 1$, where each group can contain two data streams each from a different user according to (R.1*), we can divide $\sum_{k=1}^{4} d_{k}^{[2]}=6$ data streams into $\zeta=3$ groups, as shown in Fig. 2. The precoding vectors in each group can 
(a)

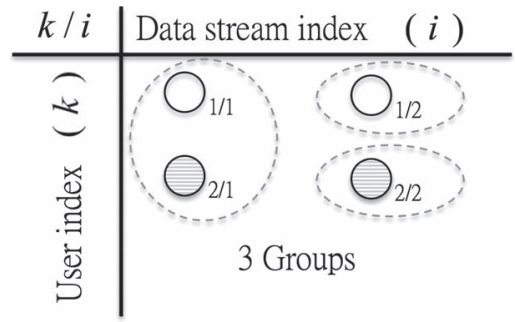

(b)

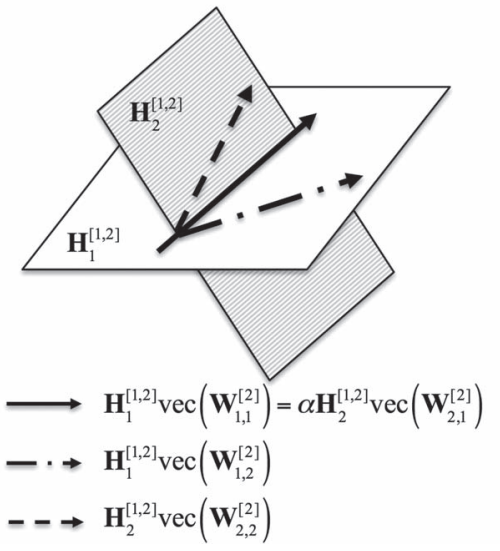

Fig. 3. An exemplary grouping by the proposed GA for a two-cell MIMO interference network with $\left(K^{[2]}, M, N, T\right)=(2,2,3,1)$ and $d_{k}^{[2]}=2, \forall k$, in Example 2. (a) A minimum number of $\zeta=3$ groups is yielded. (b) The span of the equivalent ICI channels are illustrated as two $2 \mathrm{D}$ planes in $\mathbb{R}^{3}$ whose intersection is a line, indicating that at most one equivalent ICI channel from user $(1,[2])$ can be dependent with another equivalent ICI channel from user $(2,[2])$.

be derived by (22). The solution to Problem $\mathcal{P}$ is given by $\gamma^{[1]}=\min \left\{\sum_{g=1}^{\zeta} r_{g}, N T\right\}=\min \{3,5\}=3$.

Example 2: Consider a two-cell MIMO interference network with system configurations of $\left(K^{[2]}, M, N, T\right)=(2,2,3,1)$ and $d_{k}^{[2]}=2, \forall k$. According to $\left(\mathrm{R} .1^{*}\right)$ and $\left(\mathrm{R} .2^{*}\right)$, the largest packing ratio is $2: 1$, where each group contains two data streams each from a different user. The equivalent ICI channels $\mathbf{H}_{k}^{[1,2]} \mathbf{W}_{k}^{[2]} \in \mathbb{R}^{3 \times 2}$ from user $(k,[2])$ span a two-dimensional (2D) plane in $\mathbb{R}^{3}$, for $k=1,2$. The intersection of the two 2D planes in $\mathbb{R}^{3}$ is a line, as illustrated in Fig. 3, which suggests that at most one equivalent ICI channel from user (1, [2]) can be dependent with another equivalent ICI channel from user (2, [2]). In other words, there will be at most one homogeneous group after grouping, i.e., $\psi(2,1)=1$ as shown in (R.3). Also, the following equation has a rank-one solution set:

$$
\left[\begin{array}{ll}
\mathbf{H}_{1}^{[1,2]} & \mathbf{H}_{2}^{[1,2]}
\end{array}\right]\left[\begin{array}{c}
\operatorname{vec}\left(\mathbf{W}_{1, i}^{[2]}\right) \\
\operatorname{vec}\left(\mathbf{W}_{2, j}^{[2]}\right)
\end{array}\right]=\mathbf{0}_{3 \times 1} .
$$

The nullity of matrix $\breve{\mathbf{H}}=\left[\mathbf{H}_{1}^{[1,2]}, \mathbf{H}_{1}^{[1,2]}\right]$ corresponds to the maximum number of the homogeneous groups, which is $2 M T-\operatorname{rank}(\breve{\mathbf{H}})=2 M T-N T=4-3=1$. As a result, the GA divides the data streams into $\zeta=3$ groups and the solution to Problem $\mathcal{P}$ is given by $\gamma^{[1]}=\min \left\{\sum_{g=1}^{\zeta} r_{g}, N T\right\}=$ $\min \{3,3\}=3$.

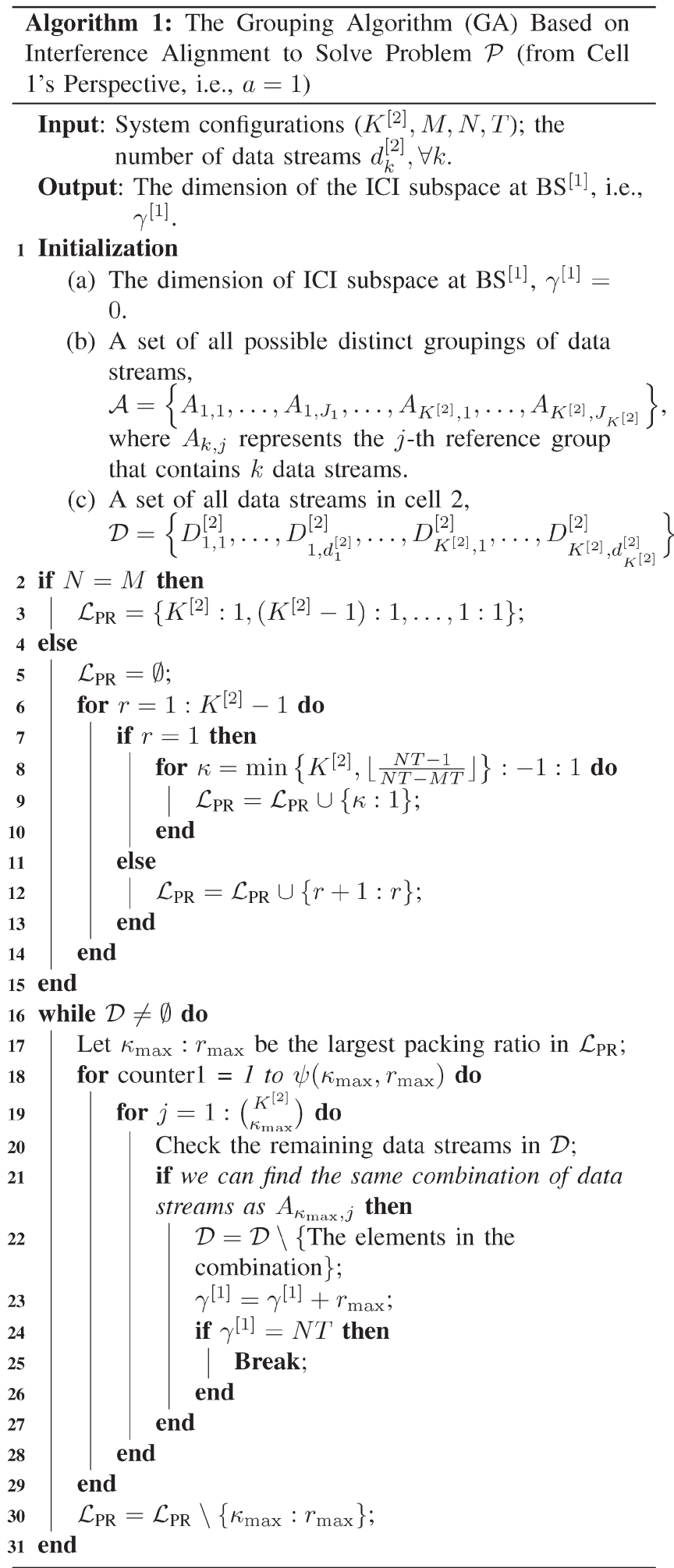

\section{B. Grouping Algorithm: General Procedure}

Based on the rules and examples presented in the previous subsection, we now describe the general procedure of the GA. The algorithm is presented in Algorithm 1 and proceeds by the following steps.

Step 1): A distinct grouping of data streams is called a reference group. The algorithm performs grouping by referencing 
(a) List of Reference Groups

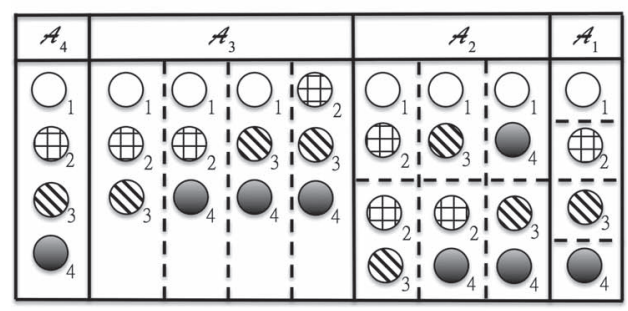

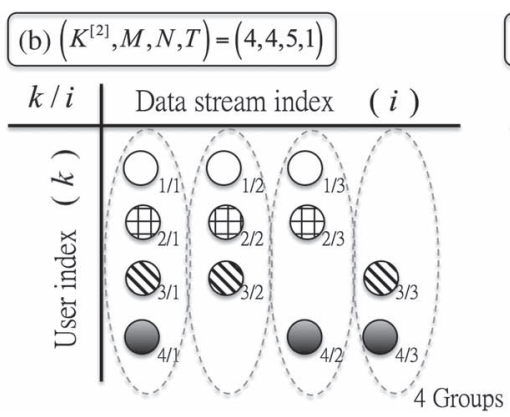

Fig. 4. An exemplary grouping by the proposed GA for a two-cell MIMO interference network with $\left(K^{[2]}, M, N\right)=(4,4,5)$ and $d_{k}^{[2]}=3, \forall k$, in Example 3 . (a) All reference groups. (b) When $T=1$, a minimum number of $\zeta=4$ groups is yielded. (c) When $T=3$, a minimum number of $\zeta=3$ groups is yielded.

the reference groups. In this initialization step, the set of reference groups, $\mathcal{A}$, is established. The set $\mathcal{A}$ can be divided into $K^{[2]}$ disjoint subsets according to the number of data streams in a group, i.e., $\mathcal{A}=\mathcal{A}_{1} \cup \mathcal{A}_{2} \cup \cdots \cup \mathcal{A}_{K^{[2]}}$. Each subset $\mathcal{A}_{k}$ is comprised of $J_{k}$ reference groups each containing $k$ data streams, i.e., $\mathcal{A}_{k}=\left\{A_{k, 1}, \ldots, A_{k, J_{k}}\right\}$, where $J_{k}=\left(\begin{array}{c}K^{[2]} \\ k\end{array}\right)$. The total number of reference groups is $|\mathcal{A}|=\left(\begin{array}{c}K^{[2]} \\ K^{[2]}\end{array}\right)+\left(\begin{array}{c}K^{[2]} \\ K^{[2]}-1\end{array}\right)+$ $\cdots+\left(\begin{array}{c}K^{[2]} \\ 1\end{array}\right)$.

Steps 2-15): All possible packing ratios are collected in the set $\mathcal{L}_{\mathrm{PR}}$ by considering $\left(\mathrm{R} .1^{*}\right)$ and $\left(\mathrm{R} .2^{*}\right)$.

Steps 16-31): The grouping begins with the largest packing ratio in $\mathcal{L}_{\mathrm{PR}}$, denoted as $\kappa_{\max }: r_{\max }$ at each iteration. Then, $\mathcal{L}_{\mathrm{PR}}$ is successively updated and grouping is successively performed in accordance with the reference groups with $\kappa_{\max }$ elements until a) all data streams have been grouped, or b) the remaining data streams are fewer than $\kappa_{\max }$ elements or the reference groups with $\kappa_{\max }$ elements have been adopted for the maximum number of $\psi\left(\kappa_{\max }, r_{\max }\right)$ times according to (R.3). In case a), the algorithm terminates and returns the solution, and in case b), the same procedure is continued on the remaining data streams with a smaller packing ratio until all data streams are grouped. Note that we set an extra stopping criterion in Steps 24-26 to avoid redundant computations. The stopping criterion occurs when the interfering signals span all $N T$-dimensional received space due to the large number of input data streams.

Example 3: Consider a two-cell MIMO interference network with system configuration of $\left(K^{[2]}, M, N, T\right)=(4,4,5,1)$ and $d_{k}^{[2]}=3, \forall k$, which gives the set of packing ratios $\mathcal{L}_{\mathrm{PR}}=$ $\{(4: 1),(3: 1),(2: 1),(3: 2),(4: 3),(1: 1)\}$. All reference groups are shown in Fig. 4(a). The grouping begins with the largest packing ratio $4: 1$ by referencing the only reference group in $\mathcal{A}_{4}$, as shown in Fig. 4(b). Since $\psi(4,1)=1$, this reference group may be adopted only once. Thus, we proceed with the second-largest packing ratio $3: 1$ and reference the groups in $\mathcal{A}_{3}$. The process repeats. Upon completion of the process, all data streams are collected into $\zeta=4$ groups. The solution to Problem $\mathcal{P}$ is given by $\gamma^{[1]}=\min \left\{\sum_{g=1}^{\zeta} r_{g}, N T\right\}=$ $\min \{4,5\}=4$. Consider a similar system configuration but with $T=3$, which gives the same set of packing ratios $\mathcal{L}_{\mathrm{PR}}$. The procedure proceeds similarly; however, since $\psi(4,1)=3$, the reference group in $\mathcal{A}_{4}$ can be adopted for a maximum of three times. This results in a smaller number of groups $(\zeta=3)$ and a different solution to Problem $\mathcal{P}\left(\gamma^{[1]}=3\right)$, as shown in Fig. 4(c). Comparing the two system configurations in this example, we can deduce that it is possible to reduce the dimension of the ICI subspace by increasing the transmission time $T$.

\section{Achievable Sum Degrees of Freedom for the Two-CEll MiMo Interference Network}

In this section, we describe the procedure to derive the achievable sum DoF for the two-cell MIMO interference network using the results of GA. Additionally, we consider the notion of the maximum achievable sum DoF under a practical condition, which can be concisely written in a closedform expression in terms of $(M, N, T)$, as opposed to the complicated form of the achievable sum DoF in terms of $\left(K^{[1]}, K^{[2]}, M, N, T\right)$.

\section{A. Algorithm for Deriving the Achievable Sum DoF}

We refer back to the GA for the derivation of the achievable sum DoF. Since data streams from the same user cannot be grouped together, it is clear that the number of groups will be greater than or equal to the maximum number of data streams from a user, i.e., $\zeta \geq \max _{k}\left\{d_{k}^{[2]}\right\}$. Also, since the packing ratio is always greater than $1: 1$, the dimension of the ICI subspace grows with the increasing number of groups according to (20). Therefore, to achieve a smaller dimension of the ICI subspace or a greater sum DoF, we should $\operatorname{make} \max _{k}\left\{d_{k}^{[2]}\right\}$ as small as possible. This suggests a "roughly equal" allocation of the total interference-free data streams among users in search for the largest achievable sum DoF. Note that exactly equal allocation may be suboptimal, since the number of the interference-free data streams for each user is a nonnegative integer and the sum DoF value may be fractional. We thus consider that the numbers of interference-free data streams only differ by one among users, i.e., $\left|d_{k}^{[2]}-d_{l}^{[2]}\right| \leq 1$ for any user $(k,[2])$ and user $(l,[2])$.

The general procedure for deriving the achievable sum DoF by using the GA is based on the idea of substituting all possible combinations of the number of data streams into the GA and selecting the combination with the greatest sum of data streams that satisfy (15b). The algorithm is presented in Algorithm 2 and proceeds by the following steps. 


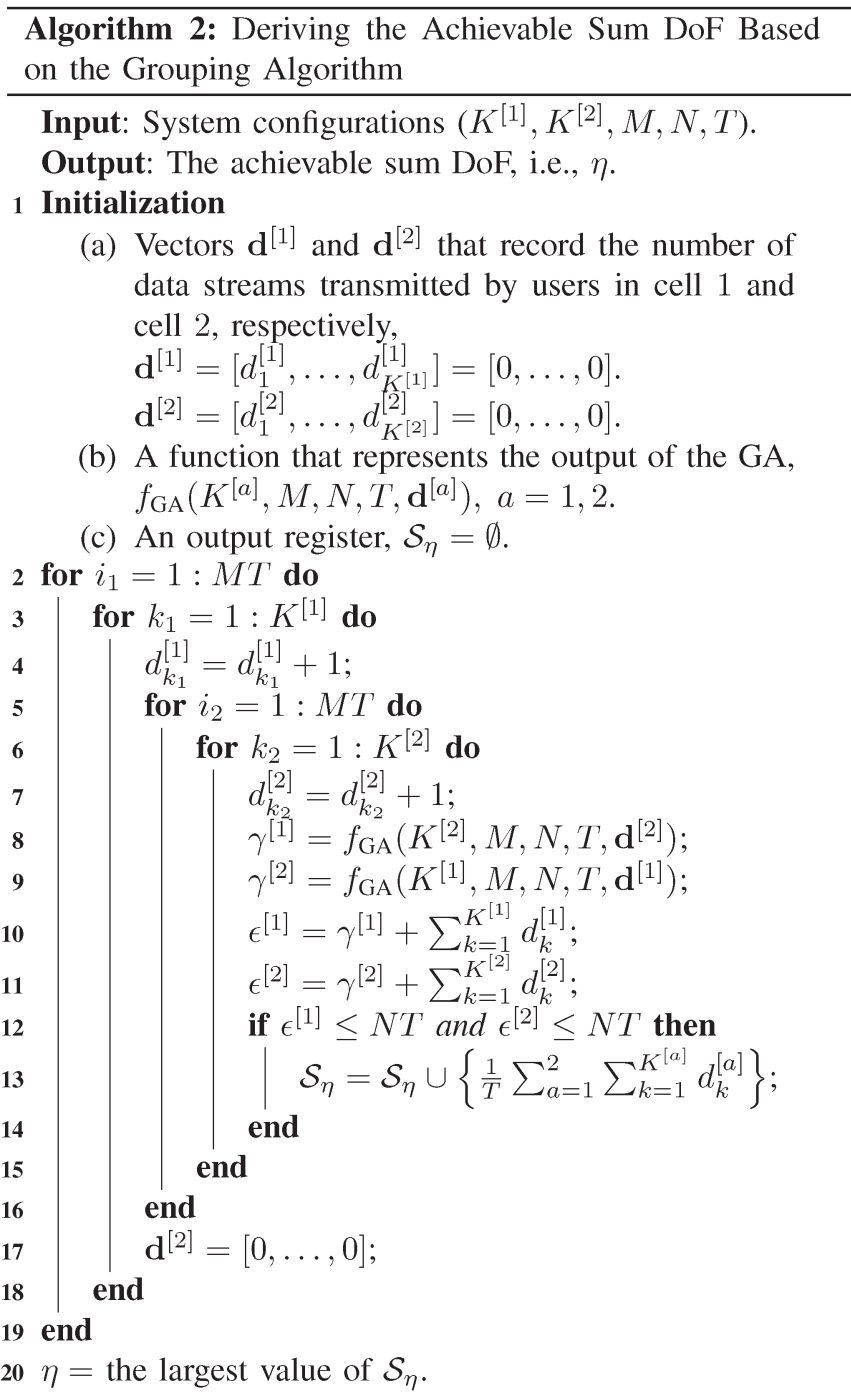

Steps 1-20): The number of data streams for each user is continuously increased up to $M T$ in a round-robin fashion. For each possible combination of the number of data streams, the GA returns a solution $\gamma^{[1]}$ (and likewise, $\gamma^{[2]}$ ). According to (15b), the dimension of the subspace spanned by both the desired and interfering signal is upper bounded by the dimension of the received signaling space, i.e., $\left(\sum_{k=1}^{K^{[a]}} d_{k}^{[a]}\right)+\gamma^{[a]} \leq N T$, for $a=1,2$. Thus, all combinations of data streams that satisfy this relationship are collected in a set (Step 13) and the largest value in the set is the achievable sum DoF (Step 20).

\section{B. Closed-Form Expression of the Maximum Achievable Sum DoF in a Practical Scenario}

As can be seen in Algorithm 2, for some given number of users in each cell, finding the achievable sum DoF involves an iterative procedure. Also, the achievable sum DoF is nondecreasing with an increasing number of users in the network. In the considered 2D-STSC scheme with a finite channel diversity of $M N T$, the number of users that can simultaneously be served and be allocated at least one dimension of interferencefree subspace is finite [37]. Here, we aim to obtain the maximum achievable sum DoF when there is the maximum number of admissible users in the network. The notion of the maximum number of admissible users specifies the maximum number of users that can be admitted to the network where each user is guaranteed at least one dimension of interference-free subspace in this finite diversity environment [38]-[40]. We obtain a closed-form expression of the maximum number of admissible users (denoted by $\mu$ ) and the maximum achievable sum DoF (denoted by $\left.\eta_{\max }\right)$, both in terms of $(M, N, T)$, without need of an iterative procedure. Note that the achievable sum DoF will remain constant at $\eta_{\max }$ after the number of users in the network reaches $\mu$.

The maximum number of admissible users can be described by

$$
\begin{aligned}
\mu \triangleq \max _{d_{k}^{[a]}, \forall k, a} & L^{[1]}+L^{[2]} \\
\text { s.t. } & d_{k}^{[a]} \geq 1, \quad \forall k, a \\
& \frac{1}{T} \sum_{a=1}^{2} \sum_{k=1}^{L^{[a]}} d_{k}^{[a]} \leq \eta\left(L^{[1]}, L^{[2]}\right)
\end{aligned}
$$

where $L^{[a]}$ represents the number of users in the $a$-th cell as a variable ${ }^{2}$ and the achievable sum DoF $\eta$ is expressed as a function of $L^{[1]}$ and $L^{[2]}$. According to the expression of the achievable sum DoF in (18), the constraint in (30) can be expressed as

$$
\left(L^{[1]}+L^{[2]}\right) \bar{d} \leq \min \left\{\left(L^{[1]}+L^{[2]}\right) M T, 2 N T-\sum_{a=1}^{2} \gamma^{[a]}\right\}
$$

or equivalently

$$
L^{[1]}+L^{[2]} \leq \frac{1}{\bar{d}}\left(2 N T-\sum_{a=1}^{2} \gamma^{[a]}\right)
$$

where $\bar{d}$ is the average number of data streams from the $L^{[1]}+$ $L^{[2]}$ users. Then, the maximum number of admissible users can also be formulated as

$$
\mu=\max _{d_{k}^{[a]} \geq 1, \forall k, a} \frac{1}{\bar{d}}\left(2 N T-\sum_{a=1}^{2} \gamma^{[a]}\right) .
$$

Note that both $\bar{d}$ and $\gamma^{[\bar{a}]}$ are related to $d_{k}^{[a]}, \forall k, a$. Clearly, from (33), to maximize the achievable $\mu$ each user can only transmit one data stream, i.e., $d_{k}^{[2]}=\bar{d}=1, \forall k$, since both $\bar{d}$ and $\gamma^{[a]}$ increase with an increased number of data streams.

Theorem 2: For a two-cell MIMO interference network with system configurations of $(M, N, T)$, the maximum achievable sum DoF is given by

$$
\eta_{\max }= \begin{cases}\frac{1}{T}\left\lfloor\frac{2 \varphi N T}{\varphi+1}\right\rfloor, & \text { if } N>M \\ \frac{2 N T-2}{T}, & \text { if } N=M\end{cases}
$$

\footnotetext{
${ }^{2}$ Notation $L^{[a]}$ is used to differentiate from notation $K^{[a]}$ which denotes the given number of users in the $a$-th cell.
} 


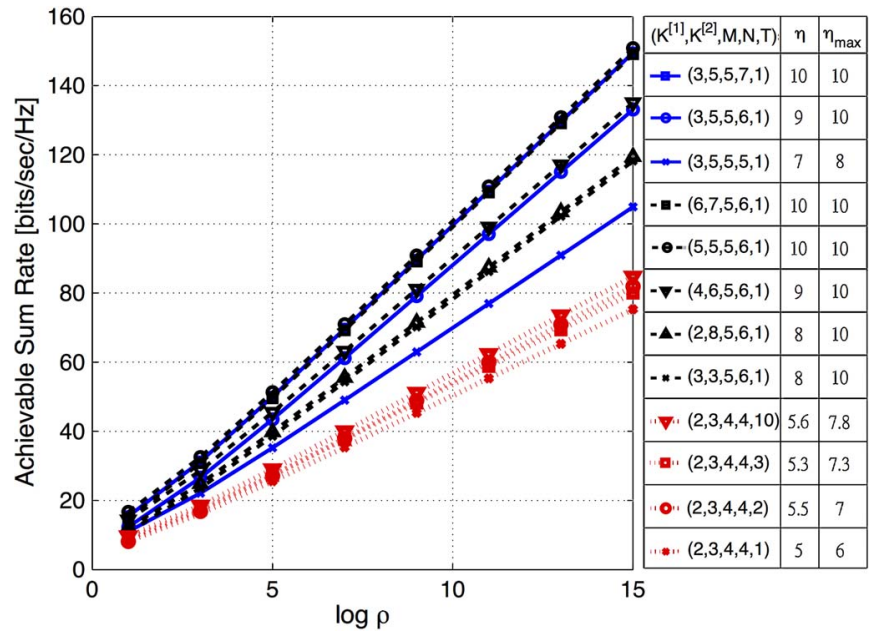

Fig. 5. The average sum rate (bits/s/Hz) at SNR $\rho$ for different system configurations of the two-cell multiuser MIMO interference network.

where

$$
\varphi=\max \left\{\left\lfloor\frac{N T-1}{N T-M T}\right\rfloor, \frac{\left\lceil\frac{N T+1}{M T}\right\rceil}{\left\lceil\frac{N T+1}{M T}\right\rceil-1}\right\} .
$$

Proof: The maximum number of admissible users can be derived by substituting $d_{k}^{[2]}=\bar{d}=1, \forall k$ into (32), i.e.,

$$
\mu=L^{[1]}+L^{[2]}=2 N T-\gamma^{[1]}-\gamma^{[2]} .
$$

Since each user transmits only one data stream, according to (R.2*) and (R.3), all groups in cell $a$ must be non-homogeneous with each other and can be constructed based on the largest packing ratio $\kappa_{\max }^{[a]}: r_{\max }^{[a]}$, where

$$
\frac{\kappa_{\max }^{[a]}}{r_{\max }^{[a]}}= \begin{cases}\max \left\{\left\lfloor\frac{N T-1}{N T-M T}\right\rfloor, \frac{\left\lceil\frac{N T+1}{M T}\right\rceil}{\left\lceil\frac{N T+1}{M T}\right\rceil-1}\right\}, & \text { if } N>M, \\ L^{[a]}, & \text { if } N=M .\end{cases}
$$

The minimum dimension of the ICI subspace in cell $a$ is thus represented by $\gamma^{[a]}=\left\lceil L^{[a]} r_{\max }^{[a]} / \kappa_{\max }^{[a]}\right\rceil$. Substituting $\gamma^{[a]}, a=$ 1,2 into (36) we have

$$
L^{[1]}+L^{[2]} \leq \begin{cases}2 N T-\frac{1}{\varphi}\left(L^{[1]}+L^{[2]}\right), & \text { if } N>M \\ 2 N T-2, & \text { if } N=M\end{cases}
$$

where $\varphi$ is defined in (35). Equivalently,

$$
L^{[1]}+L^{[2]} \leq \begin{cases}\frac{2 \varphi N T}{\varphi+1}, & \text { if } N>M \\ 2 N T-2, & \text { if } N=M .\end{cases}
$$

The maximum number of admissible users $\mu$ is derived by finding the largest integer that satisfies (39). Then, the maximum achievable sum DoF is $\eta_{\max }=(1 / T) \sum_{a=1}^{2} \sum_{k=1}^{L^{[a]}} d_{k}^{[a]}=$ $(1 / T) \sum_{a=1}^{2} \sum_{k=1}^{L^{[a]}}\{1\}=\mu / T$, which leads to (34).

\section{RESUlts AND Discussions}

Average Sum Rate vs. Log-SNR Performance: Fig. 5 illustrates the achievable sum rate vs. log-SNR performance of the proposed scheme with different system configurations. Both the achievable sum $\operatorname{DoF}(\eta)$ and the maximum achievable sum DoF $\left(\eta_{\max }\right)$ are shown beside each configuration. Configurations in the group represented by the solid, dashed, or dotted line differ only in $N,\left(K^{[1]}, K^{[2]}\right)$, or $T$, respectively. Several observations can be made. First, the achievable sum rate increases linearly with log-SNR with a slope given by the achievable sum DoF. Second, both the achievable sum rate and the achievable sum DoF increase with increasing values of $N$ or $K^{[1]}+K^{[2]}$. Third, asymmetric numbers of users may result in a different achievable sum DoF even though the total number of users in the network is the same, as shown by comparing $\left(K^{[1]}, K^{[2]}, M, N, T\right)=(5,5,5,6,1), \quad(4,6,5,6,1)$, and $(2,8,5,6,1)$. Fourth, the achievable sum DoF remains constant at $\eta_{\max }$ after the total number of users reaches the maximum number of admissible users in the network $(\mu)$, as shown by comparing $\left(K^{[1]}, K^{[2]}, M, N, T\right)=$ $(5,5,5,6,1)$ and $(6,7,5,6,1)$. For both configurations we have $\eta_{\max }=10$ and $\mu=\eta_{\max } T=10$ from Theorem 2 . Thus, both configurations overlap as they both achieve the maximum achievable sum DoF for the two-cell MIMO interference network with $(M, N, T)=(5,6,1)$. Fifth, $\eta_{\max }$ increases with an increasing $T$, as shown by comparing the last four configurations (represented by dotted lines). This is because a larger $T$ increases the channel diversity, thus allowing more admissible users in the network and yielding a larger $\eta_{\max }$ according to Theorem 2. On the other hand, $\eta$ does not increase with an increasing $T$, because $K^{[1]}$ and $K^{[2]}$ are fixed. The fractional differences in the value of $\eta$ in the four configurations are due to the division by $T$ in (8).

Comparisons of the Sum DoF: We compare the achievable sum DoF of the proposed scheme with several existing schemes described as follows. We compare our results with the combined results from [16], [17] for the achievable sum DoF, and we compare our results with the results in [3], [8], [22] adapted to the two-cell MIMO interference network for the upper bound on the DoF. Specifically, it was shown in [3] that precisely $\min \left\{N_{1}+N_{2}, M_{1}+M_{2}, \max \left\{N_{1}, M_{2}\right\}, \max \left\{N_{2}\right.\right.$, $\left.\left.M_{1}\right\}\right\}$ DoF can be achieved for a two-user interference network, where $N_{k}$ and $M_{k}$ denote the numbers of antennas for user $k$ 's receiver and transmitter, respectively. Since the two-cell MIMO interference network with $\left(K^{[1]}, K^{[2]}\right)$ users can be considered as a two-user interference network with users in each cell cooperating with each other, we can extend the result in [3] to obtain an information-theoretic upper bound on the sum DoF for the two-cell MIMO interference network, which is given by $\min \left\{2 N,\left(K^{[1]}+\right.\right.$ $\left.\left.K^{[2]}\right) M, \max \left\{N, K^{[1]} M\right\}, \max \left\{N, K^{[2]} M\right\}\right\}$. Furthermore, using the claim in [8], [22] that the precoding and decoding design will be infeasible if the number of variables is less than the number of equations, we can obtain a linear-scheme-based upper bound on the sum DoF, which is given by $\min \left\{\left(K^{[1]}+\right.\right.$ $\left.\left.K^{[2]}\right) M,\left(K^{[1]}+K^{[2]}\right)(M+N) /\left(K^{[1]}+K^{[2]}+1\right)\right\}$. Note that when $K^{[1]}=K^{[2]}=K, N=M=1$, and $T=K+1$, the proposed scheme achieves the sum DoF of $(2 K) /(K+1)$, which is equal to the IA scheme proposed in [12] for the single-antenna two-cell multiuser MIMO interference network.

Fig. 6 plots the sum DoF vs. $N$ with $\left(K^{[1]}, K^{[2]}, M\right)=(5,5$, $N-2)$. Dotted lines represent the upper bounds on the sum 


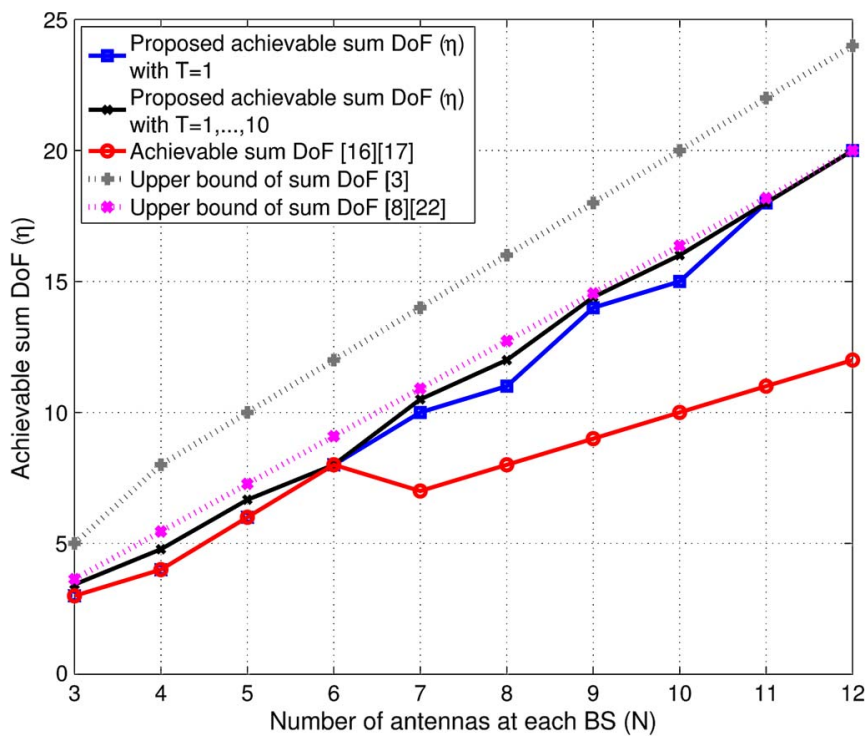

Fig. 6. Comparison of the sum DoF of the two-cell multiuser MIMO interference network with $K^{[1]}=K^{[2]}=5$ and $M=N-2$.

DoF from previous studies, and solid lines represent the achievable sum DoF derived in [16], [17] as well as by ourselves. We show our achievable sum DoF with two considerations of $T$ : i) when $T=1$, and ii) when $T=1, \ldots, 10$ where we show the maximum value obtained from these time configurations (which is similar to the spatial normalization in [18]-[20]). As compared to [16], [17], the proposed scheme achieves the same sum DoF when $N=6$ and a greater sum DoF when $N<6$ and $N>6$. Furthermore, our achievable sum DoF result with time consideration ii) approaches the linear-scheme-based upper bound derived in [8], [22] with a difference less than 1 for various values of $N$. This suggests that the proposed scheme takes nearly full advantage of the space/time dimensions.

Fig. 7 shows the sum DoF vs. $K^{[1]}=K^{[2]}=K$ with $(M, N$, $T)=(1,4,4)$. In this configuration, each user has a single antenna, which allows us to further compare our results with [14]. In [14], an information-theoretic upper bound on the sum DoF was derived, which is given by $\min \{2 K, N\}$ when $N \geq K$ and $\min \{K,((\max \{2 N, K\}) /(\max \{2 N, K\}+1)) 2 N\}$ when $N<K$. Also, in [14] an achievable sum DoF based on ZF coding scheme was derived as $\min \{N, 2 K, \max \{N, K\}\}$. As can be clearly seen in Fig. 7, our result overlaps with the information-theoretic upper bounds in [3] and [14] for $K \leq 4$, indicating that the proposed GA achieves the precise number of the sum DoF in this case. For $K>4$, the proposed scheme cannot achieve the information-theoretic upper bounds in [3] and [14], but approaches the linear-scheme-based upper bound in [8], [22] with a difference less than 1 for various values of $K$. As compared to the achievable sum DoF in [14] and in [16], [17] (note that the two achievable results overlap in this configuration), the proposed scheme achieves the same result of the sum DoF when $K \leq 4$ and a greater result when $K>4$. This figure also confirms that the achievable sum DoF remains constant at $\eta_{\max }=4.25$ (shown by the dotted horizontal line) after the number of users per cell reaches the maximum number of admissible users per cell (shown by the vertical line), i.e., when $K \geq \mu / 2=8.5$.

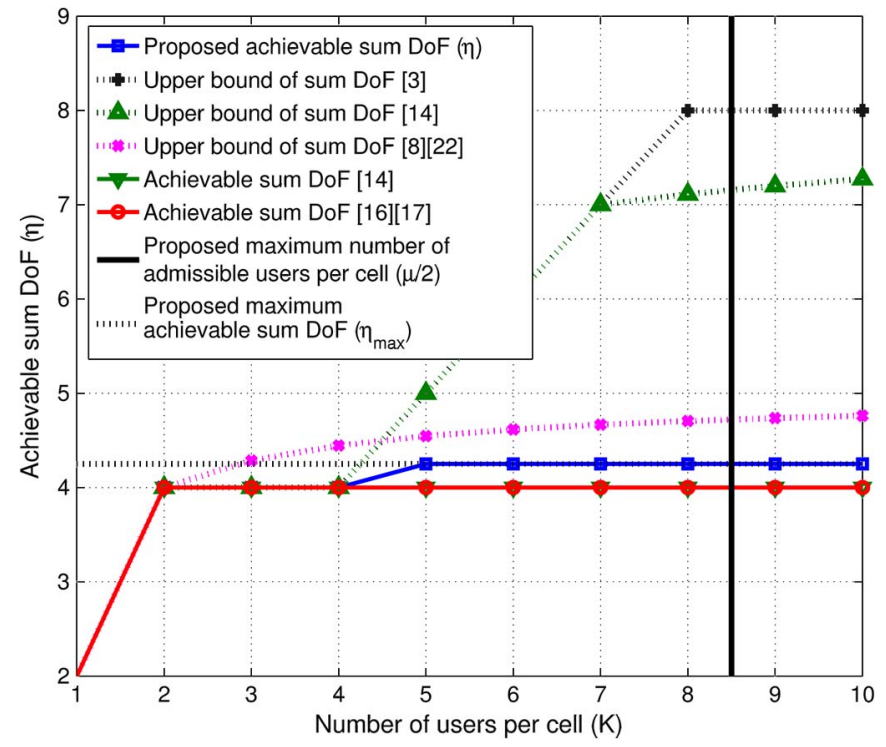

Fig. 7. Comparison of the sum DoF of the two-cell multiuser MIMO interference network with $K^{[1]}=K^{[2]}=K, M=1, N=4$, and $T=4$. This setting gives $\mu=17$.

\section{CONCLUSION}

This paper studies the sum DoF for the uplink transmission in a two-cell multiuser MIMO interference network with a finite transmission time and asymmetric numbers of users. The derivation of the achievable sum DoF is shown related to a rank minimization problem which is solved by the proposed GA. The GA corresponds to a grouping procedure that aligns the interfering data streams into a low-dimensional subspace as a group and collects as many data streams into a group with a packing ratio as large as possible. The GA provides a joint precoding and decoding scheme which is essentially a 2D-STSC. It is shown that the achievable sum DoF derived based on the GA is greater than previous results and achieves the theoretic upper bound in some cases, suggesting that the proposed scheme takes nearly full advantage of the space/time dimensions. We derive the maximum achievable sum DoF when there is the maximum number of admissible users in the network. We obtain the maximum achievable sum DoF to be $\eta_{\max }=(2 N T-2) / T$ for $N=M$ and $\eta_{\max }=(1 / T)\lfloor(2 \varphi N T) /$ $(\varphi+1)\rfloor$ for $N>M$, where $\varphi=\max \{\lfloor(N T-1) /(N T-$ $M T)\rfloor,\lceil(N T+1) /(M T)\rceil /\lceil(N T+1) /(M T)\rceil-1\}$. Since this paper assumes that all users demand for interference-free data streams, relaxing this assumption for a further analysis and design could be a worthwhile future work.

\section{APPENDIX \\ ProOF OF THEOREM 1}

We first show the necessity and then the sufficiency of the three grouping rules.

Necessity: We will show that if any of the grouping rules (R.1)-(R.3) is not satisfied, the GA will not produce a feasible solution to Problem $\mathcal{P}$.

(R.1): Suppose that $r_{g}+\alpha$ (where $\alpha \in \mathbb{N}_{+}$) data streams of user $(k,[2])$ are assigned to the same $g$-th group with packing ratio $\kappa_{g}: r_{g}$. This suggests that the dimension of the ICI 
subspace spanned by the equivalent ICI channels corresponding to these data streams is upper bounded by $r_{g}$, i.e.,

$\operatorname{rank}\left(\operatorname{col}\left\{\mathbf{H}_{k}^{[1,2]} \operatorname{vec}\left(\mathbf{W}_{k, 1}^{[2]}\right), \ldots, \mathbf{H}_{k}^{[1,2]} \operatorname{vec}\left(\mathbf{W}_{k, r_{g}+\alpha}^{[2]}\right)\right\}\right)$ $\leq r_{g}$.

Since $\mathbf{H}_{k}^{[1,2]}$ is full rank and $N T \geq r_{g}+\alpha$, (40) is equivalent to

$$
\operatorname{rank}\left(\operatorname{col}\left\{\operatorname{vec}\left(\mathbf{W}_{k, 1}^{[2]}\right), \ldots, \operatorname{vec}\left(\mathbf{W}_{k, r_{g}+\alpha}^{[2]}\right)\right\}\right) \leq r_{g}
$$

However, this violates the constraint of Problem $\mathcal{P}$ because

$$
\begin{aligned}
& \operatorname{rank}\left(\mathbf{W}_{k}^{[2]}\right)= \operatorname{rank}\left(\operatorname{vec}\left(\mathbf{W}_{k, 1}^{[2]}\right), \ldots, \operatorname{vec}\left(\mathbf{W}_{k, d_{k}^{[2]}}^{[2]}\right)\right) \\
& \stackrel{(a)}{\leq} \operatorname{rank}\left(\operatorname{vec}\left(\mathbf{W}_{k, 1}^{[2]}\right), \ldots, \operatorname{vec}\left(\mathbf{W}_{k, r_{g}+\alpha}^{[2]}\right)\right) \\
& \\
&+\operatorname{rank}\left(\operatorname{vec}\left(\mathbf{W}_{k, r_{g}+\alpha+1}^{[2]}\right), \ldots, \operatorname{vec}\left(\mathbf{W}_{k, d_{k}^{[2]}}^{[2]}\right)\right) \\
& \leq \\
& \leq r_{g}+d_{k}^{[2]}-\left(r_{g}+\alpha\right) \\
&<d_{k}^{[2]}
\end{aligned}
$$

where inequality $(a)$ holds since $\operatorname{rank}\left(\mathbf{A}_{1}, \mathbf{A}_{2}\right) \leq \operatorname{rank}\left(\mathbf{A}_{1}\right)+$ $\operatorname{rank}\left(\mathbf{A}_{2}\right)$ for arbitrary matrices $\mathbf{A}_{1}$ and $\mathbf{A}_{2}$ [41].

(R.2): When $N=M$, (R.2) degenerates to a trivial statement. When $N>M$, suppose that there are $\kappa_{g}+\alpha$ data streams in the $g$-th group, where $\kappa_{g}$ is equal to the upper bound $\left\lfloor\left(\chi_{g} M T+r_{g} N T-1\right) /(N T)\right\rfloor$ in (24) and $\alpha \in \mathbb{N}_{+}$. This results in not all precoding vectors in the solution set of $\breve{\mathbf{w}}_{g}$ being nonzero vectors, since $\breve{\mathbf{H}}_{g}$ is either a non-full-rank matrix or has no fewer rows than columns, i.e.,

$$
\begin{aligned}
\left(\kappa_{g}+\alpha-r_{g}\right) N T-\beta M T & =\left\lfloor\frac{\chi_{g} M T+r_{g} N T-1}{N T}\right\rfloor N T \\
& -\alpha N T-\beta M T-r_{g} N T \\
& \geq(\alpha-1) N T+\left(\chi_{g}-\beta\right) M T \\
& \stackrel{(a)}{\geq} 0 .
\end{aligned}
$$

where $\beta=\kappa_{g}$ when $r_{g}=1$, and $\beta=\chi_{g}$ when $r_{g}=\kappa_{g}-1$. When $r_{g}=1$, we need only to consider the case where $\chi_{g}=\kappa_{g}$ (i.e., (R.1) is satisfied), which leads to $\chi_{g}-\beta=\kappa_{g}-\kappa_{g}=0$ and thus inequality $(a)$ holds. When $r_{g}=\kappa_{g}-1$, we have $\chi_{g}-\beta=\chi_{g}-\chi_{g}=0$ and thus inequality $(a)$ also holds. Clearly, the zero precoding vectors violate the full rank constraint of Problem $\mathcal{P}$.

(R.3): Suppose that there are $\psi\left(\kappa_{h}, r_{h}\right)+\alpha$ (where $\alpha \in \mathbb{N}_{+}$) homogeneous groups after grouping, each consisting of $\kappa_{h}$ data streams. We need only to show the case where $\left(\kappa_{g}-\right.$ $\left.r_{g}\right) N T<\chi_{g} M T$ (i.e., (R.2) is satisfied). For the $g^{\prime}$-th homogeneous group, $g^{\prime}=1, \ldots, \psi\left(\kappa_{h}, r_{h}\right)+\alpha$, we can similarly define (22) with $\breve{\mathbf{H}}_{g^{\prime}}$ and $\breve{\mathbf{w}}_{g^{\prime}}$. Since these homogeneous groups are composed of data streams from the same set of users, $\operatorname{col}\left\{\breve{\mathbf{H}}_{g^{\prime}}\right\}$ is identical for all $g^{\prime}$, which is denoted by $\operatorname{col}\left\{\breve{\mathbf{H}}_{h}\right\}$ for convenience. Therefore, we have

$$
\operatorname{col}\left\{\breve{\mathbf{w}}_{1}, \ldots, \breve{\mathbf{w}}_{\psi\left(\kappa_{h}, r_{h}\right)+\alpha}\right\} \subseteq \operatorname{null}\left\{\breve{\mathbf{H}}_{h}\right\}
$$

which suggests that the rank of the space spanned by $\breve{\mathbf{w}}_{g^{\prime}}, \forall g^{\prime}$ is upper bounded by the nullity of $\breve{\mathbf{H}}_{h}$, i.e.,

$$
\begin{aligned}
\operatorname{rank} & \left(\operatorname{col}\left\{\breve{\mathbf{w}}_{1}, \ldots, \breve{\mathbf{w}}_{\psi\left(\kappa_{h}, r_{h}\right)+\alpha}\right\}\right) \\
& \leq\left(\kappa_{h} M T-\left(\kappa_{h}-r_{h}\right) N T\right)^{+} \\
& =\psi\left(\kappa_{h}, r_{h}\right) .
\end{aligned}
$$

The above condition violates the full-rank constraint of Problem $\mathcal{P}$ because

$$
\begin{aligned}
& \operatorname{rank}\left(\mathbf{W}_{k}^{[2]}\right) \\
& =\operatorname{rank}\left(\operatorname{vec}\left(\mathbf{W}_{k, 1}^{[2]}\right), \ldots, \operatorname{vec}\left(\mathbf{W}_{k, \psi\left(\kappa_{h}, r_{h}\right)+\alpha}^{[2]}\right),\right. \\
& \left.\quad \operatorname{vec}\left(\mathbf{W}_{k, \psi\left(\kappa_{h}, r_{h}\right)+\alpha+1}^{[2]}\right), \ldots, \operatorname{vec}\left(\mathbf{W}_{k, d_{k}^{[2]}}^{[2]}\right)\right) \\
& \quad \stackrel{(a)}{\leq} \operatorname{rank}\left(\operatorname{col}\left\{\breve{\mathbf{w}}_{1}, \ldots, \breve{\mathbf{w}}_{\psi\left(\kappa_{h}, r_{h}\right)+\alpha}\right\}\right) \\
& \quad+\operatorname{rank}\left(\operatorname{vec}\left(\mathbf{W}_{k, \psi\left(\kappa_{h}, r_{h}\right)+\alpha+1}^{[2]}\right), \ldots, \operatorname{vec}\left(\mathbf{W}_{k, d_{k}^{[2]}}^{[2]}\right)\right) \\
& \leq \psi\left(\kappa_{h}, r_{h}\right)+\left(d_{k}^{[2]}-\left(\psi\left(\kappa_{h}, r_{h}\right)+\alpha\right)\right) \\
& <d_{k}^{[2]}
\end{aligned}
$$

where inequality $(a)$ holds since $\operatorname{vec}\left(\mathbf{W}_{k, g^{\prime}}^{[2]}\right)$ lies in the precoding vector set of $\breve{\mathbf{w}}_{g^{\prime}}$ and $\left\{\breve{\mathbf{w}}_{g^{\prime}}\right\}_{g^{\prime}=1, \ldots, \psi\left(\kappa_{h}, r_{h}\right)+\alpha}$ is full rank.

Sufficiency: We will show that if the grouping rules (R.1)-(R.3) are all satisfied, the GA will find a feasible solution to Problem $\mathcal{P}$. Without loss of generality, we focus on the perspective of user $(k,[2]), k=1, \ldots, K^{[2]}$.

Suppose that all data streams are divided into $\zeta$ groups, denoted as $\mathcal{G}_{1}, \ldots, \mathcal{G}_{\zeta}$. The precoding vectors in the $g$-th group can be derived by solving $\breve{\mathbf{w}}_{g}$ in (22). To show that the precoding vectors obtained from (22) are feasible, we consider two data streams, i.e., the $i$-th and $j$-th data stream of user $(k,[2])$ (where $1 \leq i, j \leq d_{k}^{[2]}$ and $i \neq j$ ), denoted by $D_{k, i}^{[2]} \in \mathcal{G}_{i}$ and $D_{k, j}^{[2]} \in \mathcal{G}_{j}$, which belong to the $i$-th and $j$-th groups, respectively. We consider two cases separately.

1) $i$-th Group and the $j$-th Group are Homogeneous: In this case, two groups contain the same number of data streams and have the same packing ratio, i.e., $\kappa_{i}=\kappa_{j}$ and $r_{i}=r_{j}$. Also, since $\breve{\mathbf{H}}_{i}$ and $\breve{\mathbf{H}}_{j}$ are composed of the channels from the same set of users, we have $\operatorname{col}\left\{\breve{\mathbf{H}}_{i}\right\}=\operatorname{col}\left\{\breve{\mathbf{H}}_{j}\right\}$, and the corresponding precoding vectors have the same solution set, i.e.,

$$
\begin{aligned}
& \operatorname{col}\left\{\breve{\mathbf{w}}_{i}, \breve{\mathbf{w}}_{j}\right\}=\operatorname{col}\left\{\left[\cdots\left(\operatorname{vec}\left(\mathbf{W}_{k, i}^{[2]}\right)\right)^{\top} \cdots\right]^{\top},\right. \\
& {\left.\left[\cdots\left(\operatorname{vec}\left(\mathbf{W}_{k, j}^{[2]}\right)\right)^{\top} \cdots\right]^{\top}\right\} } \\
&= \operatorname{null}\left\{\breve{\mathbf{H}}_{i}\right\}=\operatorname{null}\left\{\breve{\mathbf{H}}_{j}\right\} .
\end{aligned}
$$

Since (R.3) is satisfied and two groups are homogeneous, we have $\psi\left(\kappa_{i}, r_{i}\right) \geq 2$. Also, the nullity of $\breve{\mathbf{H}}_{i}$ is upper bounded 
by $\psi\left(\kappa_{i}, r_{i}\right)$, as shown in (45). Therefore, we can always design two independent precoding vectors from (22), i.e., $\operatorname{rank}\left(\operatorname{col}\left\{\breve{\mathbf{w}}_{i}, \breve{\mathbf{w}}_{j}\right\}\right)=2$. In addition, since (R.1) and (R.2) are satisfied, any precoding vector derived from (22) is a nonzero vector, which suggests $>$

$\operatorname{rank}\left(\operatorname{col}\left\{\breve{\mathbf{w}}_{i}, \breve{\mathbf{w}}_{j}\right\}\right)$

$$
=\operatorname{rank}\left(\operatorname{col}\left\{\operatorname{vec}\left(\mathbf{W}_{k, i}^{[2]}\right), \operatorname{vec}\left(\mathbf{W}_{k, j}^{[2]}\right)\right\}\right) \text {. }
$$

Thus, we have two independent precoding vectors for user $(k,[2])$.

2) $i$-th Group and the $j$-th Group are not Homogeneous: Denote null $\left\{\breve{\mathbf{H}}_{i}\right\}$ and null $\left\{\breve{\mathbf{H}}_{j}\right\}$ as the solution set of the precoding vectors of the $i$-th and $j$-th group, respectively. We discuss two subcases separately: i) null $\left\{\breve{\mathbf{H}}_{i}\right\} \subseteq \operatorname{null}\left\{\breve{\mathbf{H}}_{j}\right\}$ (or $\left.\operatorname{null}\left\{\breve{\mathbf{H}}_{j}\right\} \subseteq \operatorname{null}\left\{\breve{\mathbf{H}}_{i}\right\}\right)$, and ii) $\operatorname{null}\left\{\breve{\mathbf{H}}_{i}\right\} \nsubseteq \operatorname{null}\left\{\breve{\mathbf{H}}_{j}\right\}$.

Subcase i) corresponds to the situation where the users in the $i$-th group are a subset of the users in the $j$-th group. Thus, we can always design two independent precoding vectors for user $(k,[2])$ as long as $\breve{\mathbf{w}}_{i} \subseteq \operatorname{null}\left\{\breve{\mathbf{H}}_{i}\right\}$ and $\breve{\mathbf{w}}_{j} \subseteq \operatorname{col}\left\{\operatorname{null}\left\{\breve{\mathbf{H}}_{j}\right\} \backslash\right.$ $\left.\operatorname{null}\left\{\breve{\mathbf{H}}_{i}\right\}\right\}$, where $\operatorname{rank}\left(\operatorname{col}\left\{\operatorname{null}\left\{\breve{\mathbf{H}}_{j}\right\} \backslash \operatorname{null}\left\{\breve{\mathbf{H}}_{i}\right\}\right\}\right)=\left(\kappa_{j}-\right.$ $\left.\kappa_{i}\right) M T$ is positive since $\kappa_{j}>\kappa_{i}$ always holds in this case.

Subcase ii) corresponds to the situation where the coding structures of two precoding vectors corresponding to data streams $D_{k, i}^{[2]}$ and $D_{k, j}^{[2]}$ are different. As a result, the two precoding vectors are independent, i.e.,

$$
\operatorname{rank}\left(\operatorname{vec}\left(\mathbf{W}_{k, i}^{[2]}\right), \operatorname{vec}\left(\mathbf{W}_{k, j}^{[2]}\right)\right)=\operatorname{rank}\left(\breve{\mathbf{w}}_{i}, \breve{\mathbf{w}}_{j}\right)=2
$$

where $\breve{\mathbf{w}}_{i} \subseteq \operatorname{null}\left\{\breve{\mathbf{H}}_{i}\right\}$ and $\breve{\mathbf{w}}_{j} \subseteq \operatorname{null}\left\{\breve{\mathbf{H}}_{j}\right\}$.

The above strategy can be generalized to more than two data streams of user $(k,[2])$ to show independency for the corresponding precoding vectors. In summary, we can always find $d_{k}^{[2]}$ independent precoding vectors from the following precoding set when (R.1)-(R.3) are all satisfied:

$$
\begin{array}{r}
\mathcal{W}_{k}=\left\{\mathbf{W}_{k}^{[2]} \in \mathbb{R}^{M T \times d_{k}^{[2]}} \mid \operatorname{rank}\left(\mathbf{W}_{k}^{[2]}\right)=d_{k}^{[2]}, D_{k, i}^{[2]} \in \mathcal{G}_{g},\right. \\
\left.i=1, \ldots, d_{k}^{[2]}, g=1, \ldots, \zeta\right\} .
\end{array}
$$

\section{REFERENCES}

[1] M. A. Maddah-Ali, A. S. Motahari, and A. K. Khandani, "Signaling over MIMO multi-base systems: Combination of multi-access and broadcast schemes," in Proc. IEEE ISIT, Seattle, WA, USA, Jul. 2006, pp. 2104-2108.

[2] V. R. Cadambe and S. A. Jafar, "Interference alignment and degrees of freedom of the $K$-user interference channel," IEEE Trans. Inf. Theory, vol. 54, no. 8, pp. 3425-2441, Aug. 2008.

[3] S. A. Jafar and M. J. Fakhereddin, "Degrees of freedom for the MIMO interference channel," IEEE Trans. Inf. Theory, vol. 53, no. 7, pp. 26372642, Jul. 2007.

[4] T. Gou and S. A. Jafar, "Degrees of freedom of the $K$-user $M \times N$ MIMO interference channel," IEEE Trans. Inf. Theory, vol. 56, no. 12, pp. 6040-6057, Dec. 2010.

[5] A. Ghasemi, A. S. Motahari, and A. K. Khandani, "Interference alignment for the K-user MIMO interference channel," in Proc. IEEE ISIT, Austin, TX, USA, Jun. 2010, pp. 360-364.

[6] K. Gomadam, V. Cadambe, and S. A. Jafar, "Approaching the capacity of wireless networks through distributed interference alignment," in Proc. IEEE GLOBECOM, New Orleans, LA, USA, Dec. 2008, pp. 1-6.
[7] S. W. Peters and R. W. Heath, "Interference alignment via alternating minimization," in Proc. IEEE ICASSP, Taipei, Taiwan, Apr. 2009, pp. 2445-2448.

[8] C. M. Yetis, T. Gou, S. A. Jafar, and A. H. Kayran, "On feasibility of interference alignment in MIMO interference networks," IEEE Trans. Signal Process., vol. 58, no. 9, pp. 4771-4782, Sep. 2010.

[9] C. M. Yetis, T. Gou, S. A. Jafar, and A. H. Kayran, "Feasibility conditions for interference alignment," in Proc. IEEE GLOBECOM, Honolulu, HI, USA, Nov. 2009, pp. 1-6.

[10] B. Nosrat-Makouei, J. G. Andrews, and R. W. Heath, "A simple SINR characterization for linear interference alignment over uncertain MIMO channels," in Proc. IEEE ISIT, Austin, TX, USA, Jun. 2010, pp. 2288-2292.

[11] S. A. Jafar and S. Shitz, "Degrees of freedom region of the MIMO X channel channel," IEEE Trans. Inf. Theory, vol. 54, no. 1, pp. 151-170, Jan. 2008.

[12] C. Suh and D. Tse, "Interference alignment for cellular networks," in Proc. 46th Annu. Conf. Commun., Control Comput., Monticello, IL, USA, Sep. 2008, pp. 1037-1044.

[13] S.-H. Park and I. Lee, "Analysis of degrees of freedom of interfering MISO broadcast channels," in Proc. IEEE GLOBECOM, Honolulu, HI, USA, Nov. 2009, pp. 1-6.

[14] S.-H. Park and I. Lee, "Degrees of freedom for mutually interfering broadcast channels," IEEE Trans. Inf. Theory, vol. 58, no. 1, pp. 393-402, Jan. 2012

[15] J. Sun, Y. Liu, and G. Zhu, "On the degrees of freedom of the cellular network," in Proc. IEEE ICC, Cape Town, South Africa, May 2010, pp. 1-5.

[16] J. Kim, S.-H. Park, H. Sung, and I. Lee, "Sum rate analysis of two-cell MIMO broadcast channels: Spatial multiplexing gain," in Proc. IEEE ICC, Cape Town, South Africa, May 2010, pp. 1-5.

[17] W. Shin, N. Lee, J. B. Lim, C. Shin, and K. Jang, "On the design of interference alignment scheme for two-cell MIMO interfering broadcast channels," IEEE Trans. Wireless Commun., vol. 10, no. 2, pp. 437-442, Feb. 2011.

[18] G. Sridharan and W. Yu, "Degrees of freedom of MIMO cellular networks with two cells and two users per cell," in Proc. IEEE ISIT, Istanbul, Turkey, Jul. 2013, pp. 1774-1778.

[19] G. Sridharan and W. Yu, "Degrees of freedom of MIMO cellular networks: Two-cell three-user-per-cell case," in Proc. IEEE GLOBECOM, Atlanta, GA, USA, Dec. 2013, pp. 1968-1973.

[20] C. Wang, T. Gou, and S. A. Jafar, "Subspace alignment chains and the degrees of freedom of the three-user MIMO interference channel," in Proc. IEEE ISIT, Cambridge, MA, USA, Jul. 2012, pp. 2471-2475.

[21] C. Wang, H. Sun, and S. A. Jafar, "Genie chains and the degrees of freedom of the K-user MIMO interference channel," in Proc. IEEE ISIT, Cambridge, MA, USA, Jul. 2012, pp. 2476-2480.

[22] Y. Ma, J. Li, R. Chen, and Q. Liu, "On feasibility of interference alignment for $l$-cell constant cellular interfering networks," IEEE Commun. Lett. vol. 16, no. 5, pp. 714-716, May 2012.

[23] T. M. Cover and J. A. Thomas, Elements of Information Theory, 2nd ed. Hoboken, NJ, USA: Wiley, Jul. 2006.

[24] R. G. Gallager, Information Theory and Reliable Communication. Hoboken, NJ, USA: Wiley, Jan. 1968.

[25] G. Bresler, D. Cartwright, and D. Tse, "Feasibility of interference alignment for the MIMO interference channel: The symmetric square case," in Proc. IEEE ITW, Paraty, Brazil, Oct. 2011, pp. 447-451.

[26] M. Razaviyayn, G. Lyubeznik, and Z.-Q. Luo, "On the degrees of freedom achievable through interference alignment in a MIMO interference channel," IEEE Trans. Signal Process., vol. 60, no. 2, pp. 812-821, Feb. 2012.

[27] D. Gesbert et al., "Multi-cell MIMO cooperative networks: A new look at interference," IEEE J. Sel. Areas Commun., vol. 28, no. 9, pp. 1380-1408, Dec. 2010.

[28] R. Doostnejad, T. J. Lim, and E. S. Sousa, "Space-time spreading codes for a multiuser MIMO system," in Conf. Rec. 36th Asilomar Conf. Signals, Syst. Comput., Pacific Grove, CA, USA, Nov. 2002, vol. 2, pp. 1374-1378.

[29] G. Bresler and D. Tse, "3 user interference channel: Degrees of freedom as a function of channel diversity," in Proc. 47th Annu. Allerton Conf. Commun., Control, Comput., Monticello, IL, USA, Sep. 2009, pp. 265-271.

[30] J. Kim, S.-H. Park, H. Sung, and I. Lee, "Spatial multiplexing gain for two interfering MIMO broadcast channels based on linear transceiver," IEEE Trans. Wireless Commun., vol. 9, no. 10, pp. 3012-3017, Oct. 2010.

[31] Q. Shi, M. Razaviyayn, Z.-Q. Luo, and C. He, "An iteratively weighted MMSE approach to distributed sum-utility maximization for a MIMO 
interfering broadcast channel," IEEE Trans. Signal Process., vol. 59, no. 9, pp. 4331-4340, Sep. 2011.

[32] D. Tse and P. Viswanath, Fundamentals of Wireless Communication. Cambridge, U.K.: Cambridge Univ. Press, 2005.

[33] D. S. Papailiopoulos and A. G. Dimakis, "Interference alignment as a rank constrained rank minimization," IEEE Trans. Signal Process., vol. 60, no. 8, pp. 4278-4288, Aug. 2012.

[34] M. Mesbahi and G. P. Papavassilopoulos, "On the rank minimization problem over a positive semidefinite linear matrix inequality," IEEE Trans. Autom. Control, vol. 42, no. 2, pp. 239-243, Feb. 1997.

[35] B. Recht, M. Fazel, and P. Parrilo, "Gaurateed minimum-rank solutions of linear matrix equations via nuclear norm minimization," in 45th Annиal Allerton Conf., Monticello, IL, USA, Sep. 2007, pp. 42-48.

[36] M. Fazel, H. Hindi, and S. Boyd, "Rank minimization and applications in system theory," in Proc. Amer. Control Conf., Boston, MA, USA, Jun. 2004, pp. 3273-3278.

[37] L. Li, H. Jarfarkhani, and S. A. Jafar, "Towards the feasibility conditions for linear interference alignment with symbol extensions: A diversity constraint," in Proc. IEEE GLOBECOM, Anaheim, CA, USA, Dec. 2012, pp. 2328-2333.

[38] L. J. Cimini, G. J. Foschini, and L. A. Shepp, "Single-channel usercapacity calculations for self-organizing cellular systems," IEEE Trans. Commun., vol. 42, no. 12, pp. 3137-3143, Dec. 1994.

[39] D. Tse and S. Hanly, "Linear multiuser receivers: Effective interference, effective bandwidth and user capacity," IEEE Trans. Inf. Theory, vol. 45, no. 2, pp. 641-657, Mar. 1999.

[40] P. Viswanath, V. Anantharam, and D. Tse, "Optimal sequences, power control, and user capacity of synchronous CDMA systems with linear MMSE multiuser receivers," IEEE Trans. Inf. Theory, vol. 45, no. 6, pp. 1968-1983, Sep. 1999.

[41] L. E. Spence, A. J. Insel, and S. H. Friedberg, Elementary Linear Algebra: A Matrix Approach, 2nd ed. Upper Saddle River, NJ, USA: PrenticeHall, Jun. 2007.

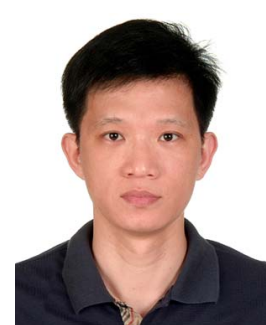

Che-Chen Chou (S'09) received the B.S. degree in electrical engineering from National Cheng Kung University, Tainan, Taiwan; the M.S. degree in electronics engineering from National Taiwan University of Science and Technology, Taipei, Taiwan; and the $\mathrm{Ph} . \mathrm{D}$. degree in communications engineering from National Tsing Hua University, Hsinchu, Taiwan. He has been in the IC design industry for more than 18 years. He is currently with Mediatek as a leader of several 4G smart phone IC projects. His interests lie in IC design and signal processing in communication

systems.

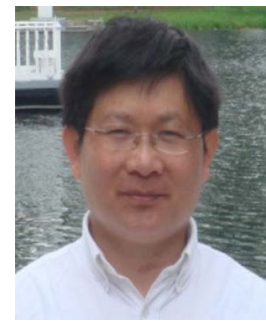

Jen-Ming Wu (M'98) received the B.S. degree from National Taiwan University, Taipei, Taiwan, in 1988; the M.S. degree from Polytechnic Institute of New York University, Brooklyn, NY, USA, in 1991; and the Ph.D. degree from the University of Southern California, Los Angeles, CA, USA, in 1998, all in electrical engineering. From 1998 to 2003, he was with Sun Microsystems Inc., Sunnyvale, CA, USA, as Member of Technical Staff. Since 2003, he has been with the faculty of the Institute of Communications Engineering and the Department of Electrical Engineering, National Tsing Hua University, Hsinchu, Taiwan, where he is currently an Associate Professor. He has worked on various fields of electrical engineering from theory to practice. His research interests include communication transmission and detection, MIMO network interference management, MIMO cognitive radio processing, MIMO signal processing and transceiver design, and high-speed interface tranceiver design.

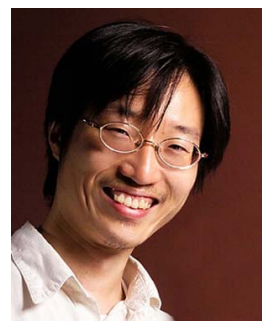

Ronald Y. Chang (M'12) received the B.S. degree in electrical engineering from National Tsing Hua University, Hsinchu, Taiwan, in 2000; the M.S. degree in electronics engineering from National Chiao Tung University, Hsinchu, in 2002; and the Ph.D. degree in electrical engineering from the University of Southern California, Los Angeles, CA, USA, in 2008. From 2002 to 2003, he was with the Industrial Technology Research Institute, Hsinchu. In 2008 , he was a research intern at the Mitsubishi Electric Research Laboratories, Cambridge, MA, USA. Since 2010, he has been with the Research Center for Information Technology Innovation, Academia Sinica, Taipei, Taiwan, where he is currently an Assistant Research Fellow. He has published about 50 papers and has four granted U.S. patents. His research interests include wireless communications and networking. He was an Exemplary Reviewer for the IEEE COMMUNICATIONS LETTERS in 2012 and a recipient of the Best Paper Award from the IEEE Wireless Communications and Networking Conference 2012. He has contributed to various conferences as a Technical Program Committee Member, including the IEEE International Conference on Communications 2012, 2013, and 2015. 